\title{
Total Resection of Complex Spinal Cord Lipomas: How, Why, and When to Operate?
}

\author{
Dachling PANG ${ }^{1,2}$ \\ ${ }^{1}$ Regional Centre of Pediatric Neurosurgery, Kaiser Permanente Medical Center, Oakland, \\ California, USA; \\ ${ }^{2}$ Department of Neurological Surgery, University of California, Davis, Sacramento, \\ California, USA
}

\begin{abstract}
This article shows the long-term advantage of total resection of complex spinal cord lipomas over partial resection and over non-surgical treatment for children with asymptomatic lipomas. The classification, embryogenesis, and technique of total resection of complex lipomas are described. The 20-year outcome of 315 patients who had total resection is measured by overall progression-free survival (PFS, Kaplan-Meier), and by subgroup Cox multivariate hazard analysis for the influence of four variables: lipoma type, symptoms, prior surgery, and post-operative cord-sac ratio. These results are compared to 116 patients who underwent partial resection, and to two published series of asymptomatic lipomas followed without surgery. The PFS after total resection for all lipomas is $88.1 \%$ over 20 years vs. $34.6 \%$ for partial resection at 10.5 years $(p<0.0001)$. The PFS for total resection of asymptomatic virgin lipomas rose to $98.8 \% \mathrm{vs.} 60 \%$ and $67 \%$ for non-surgical treatment. Partial resection also compares poorly to non-surgical treatment for asymptomatic lipomas. Multivariate analyses show that a low cord-sac ratio is the only independent variable that predicts good outcome. Pre-operative profiling shows the ideal patient for total resection is a young child with a virgin asymptomatic lipoma, who, with a PFS of $99.2 \%$, is essentially cured. The technique of total resection can be learned by any neurosurgeon. Its long-term protection against symptomatic recurrence is better than partial resection and conservative management. The surgery should be done at diagnosis, except for asymptomatic small infants in whom surgery should be postponed till 6 months to minimize morbidity.
\end{abstract}

Key words: embryogenesis, outcome, spinal lipoma, surgery

Partial resection of complex spinal cord lipomas is associated with a high rate of symptomatic recurrence due to retethering. ${ }^{1)}$. The author and his colleagues have amply documented this in their previous publications. ${ }^{1-3)}$ Since 1991, we have performed over 360 total/near-total resections of complex lipomas with radical reconstruction of the neural placodes, designed to minimize the pre-conditions of retethering. Twenty years of follow-up have proven the long-term benefits of this technique. ${ }^{1,2)}$ I now strongly advocate for total resection of spinal cord lipomas and radical reconstruction of the neural placode over partial resection because aggressive surgery, contrary to traditional teaching, is safe and gives far better long-term progression-free survival (PFS). ${ }^{1)}$

The rationale for total lipoma resection is based on three hypotheses: (1) the high rate of symptomatic recurrence after partial resection is due to re-tethering;

Received December 18, 2014; Accepted February 17, 2015
(2) re-tethering in partial resection is promoted by three factors: a tight content-container relationship between spinal cord and dural sac, a large "sticky" raw surface of residual fat, and incomplete detachment of the terminal neural placode from residual lipoma; and (3) total resection can eliminate the factors conducive to re-tethering and thus reduces the probability of symptomatic recurrence.

The objective of surgery is therefore to create conditions that will minimize re-tethering. The first condition predicates on the fact that the normal spinal cord exhibits intradural motions to gravity and postural changes on ultrasonography and dynamic imaging. ${ }^{4,5)}$ Reducing the content-container ratio and amplifying the degree of freedom of the cord within the dural sac must lessen re-sticking by limiting sustained contact between cord and dura, this sustained contact being intuitively a necessary condition preceding the formation of fibrous adhesions. To do this, the cord bulk must be drastically reduced. 
For large rambling virgin lipomas, this means resection of all or most of the fat down to the thin, supple neural placode. For redo lipomas, the hard, grasping cicatrix must also be removed. The ultimate aim is to render the thinnest, most pliant neural placode possible that can be atraumatically neurulated (sutured pia-to-pia; see below) without distortion or strangulation to form a slender, round tube. [The term neural placode in lipoma is borrowed from the familiar essential element of an open neural tube defect or ONTD, to emphasize its equivalent "neural" nature once purified of fat. The synonymous usage of the term in lipoma and ONTD is logical if one ponders the embryogenesis of the two entities (see below): the "placode" in each case represents the original embryonic neural plate blighted in its final completing process, one invaded by paraxial mesenchyme, the other thwarted in its midline dorsal fusion.] The raw, sticky lipoma bed is simultaneously concealed within the tube and the sac is enlarged by a capacious dural graft. Finally, total resection also increases the chances of terminal untethering.

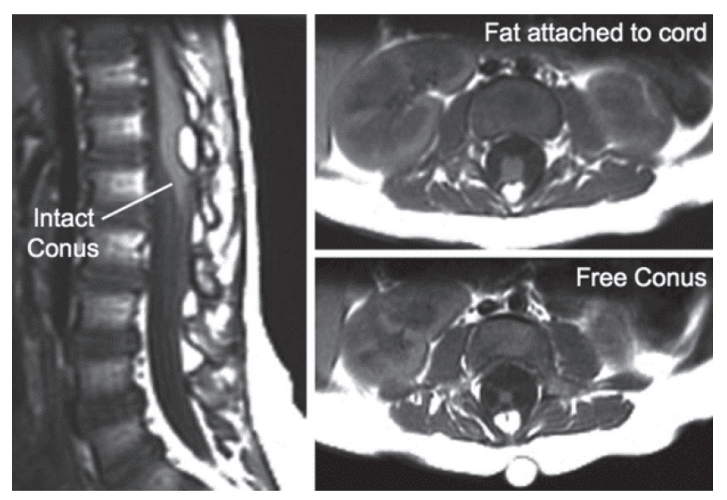

Fig. 1 Dorsal lipoma on magnetic resonance imaging. Sagittal image shows intact conus caudal to lipoma stalk. Axial images: upper shows site of lipoma attachment to cord; lower shows free conus just caudal to the level of lipoma attachment.

\section{Anatomy and Classification}

In the literature, the nomenclature of spinal cord lipoma is imprecise and inconsistent. Here, we are defining the types of lipomas as follows:

\section{Dorsal lipoma}

The lipoma-cord interface is entirely on the dorsal surface of the lumbar spinal cord, always sparing the distal conus (Fig. 1). The junctional demarcation between lipoma, cord, and pia, the fusion line (see below), can always be traced neatly along a roughly oval track, separating fat from the dorsal root entry zone (DREZ) and dorsal nerve roots laterally (Fig. 2). The lipoma therefore never contains nerve roots. The lipomatous stalk runs through an equally discrete dorsal dural defect to blend with extradural fat. The uninvolved conus often ends in a thickened filum terminale.

\section{Transitional lipoma}

The rostral portion of this type is identical to that of a dorsal lipoma, with a discrete fusion line and easily identifiable DREZ and dorsal roots. Unlike the dorsal type, however, which always spares the conus, the transitional lipoma then plunges caudally to involve the conus as the plane of the fusion line cuts ventrally and obliquely towards the tip of the conus likened to making a slanting, bevelled cut on a stick (Fig. 3A). The lipoma-cord interface thus created may be undulating and tilted so that the neural placode is rotated to one side or even spun into a parasagittal edge-on orientation, but the neural tissue is always ventral to this interface, i.e., on the side of the nerve roots exit, and the DREZ and the nerve roots are predictably localizable lateral and ventral to the fusion line and therefore do not course through the fat (Fig. 3B). There may or may not be a discrete filum. The dorsal dural defect extends to the caudal end of the thecal sac and may be much larger on the side where an asymmetric lipoma is dominant.

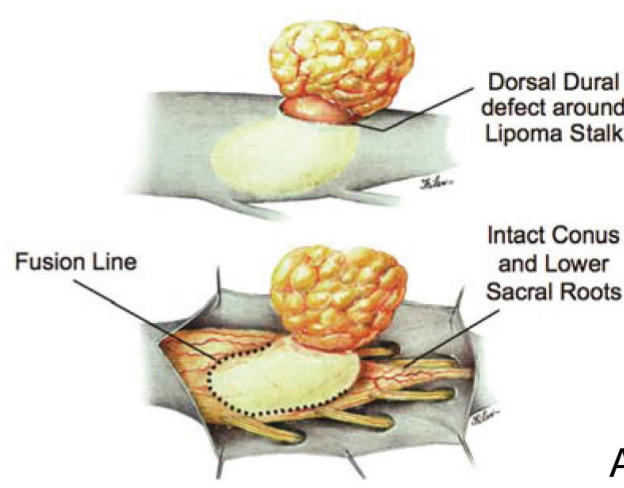

A

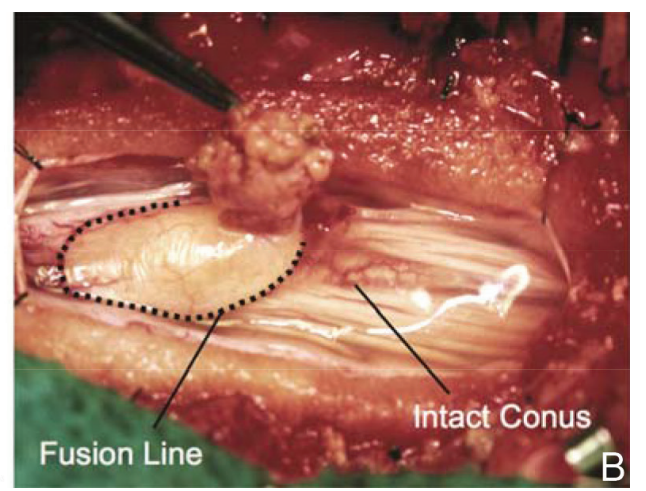

Fig. 2 Dorsal lipoma. A: Intraoperative drawings show neat dorsal dural defect through which lipoma stalk enters. Lower shows circumferential fusion line and intact conus. B: Intraoperative picture shows neat oval fusion line around lipoma-cord interface on a horizontal plane. Note intact conus and caudal sacral roots. 


\section{Terminal lipoma}

Unlike the dorsal and transitional types, terminal lipomas insert into the caudal extremity of the conus without blending with the spinal cord or its root entry zones. All the sacral roots unmistakably leave the conus rostral to the lipoma, and in most cases the conus itself looks normal. The dural sac and the dorsal myofascial coverings are intact. The lipoma either replaces the filum entirely or is separated from the conus tip by a short, thickened filum.

The surgery for terminal lipoma is relatively straight forward and is therefore beyond the scope of this review.

\section{Chaotic lipoma}

This novel type is so named because it does not "follow the rules" of either the dorsal, transitional,


Fig. 3 Transitional lipoma. A: Left: Sagittal magnetic resonance imaging shows lipoma begins dorsally but involves entire conus. Ventral side of neural placode is free of fat. Right: The plane of the fusion line begins dorsally then cuts obliquely towards the tip of the conus. The array of DREZ and dorsal roots is also forced to slant dorso-ventrally. B: Intraoperative picture showing massive lipoma but very distinct dorso-ventral fusion line separating fat from the DREZ and dorsal roots, which always lie lateral and ventral to the fusion line. The ventral side of the placode is always free of fat in a regular transitional lipoma. or terminal lipoma. It begins dorsally in an orderly fashion as in a dorsal or transitional lipoma, but its caudal portion is ventral to the neural placode and does engulf neural tissue and nerve roots (Fig. 4A). The fusion line may be distinct rostrally but quickly becomes blurred distally, and the locations of the DREZ and nerve roots are less predictable. The moniker "chaotic" depicts the sometimes confusing blend of the ventral fat and neural placode, and the often impossible task of separating fat from neural tissue at surgery (Fig. 4B). Chaotic lipomas are uncommon but are characteristically seen with sacral agenesis. ${ }^{2)}$

The literature ${ }^{6,7)}$ mentions one other lipoma type, the lipomyelomeningocele, in which part of the distal conus extends into the extraspinal compartment, dragging with it a small collar of dural sac (Fig. 5). The basic structure is either that of a transitional or a dorsal lipoma. Accordingly, we
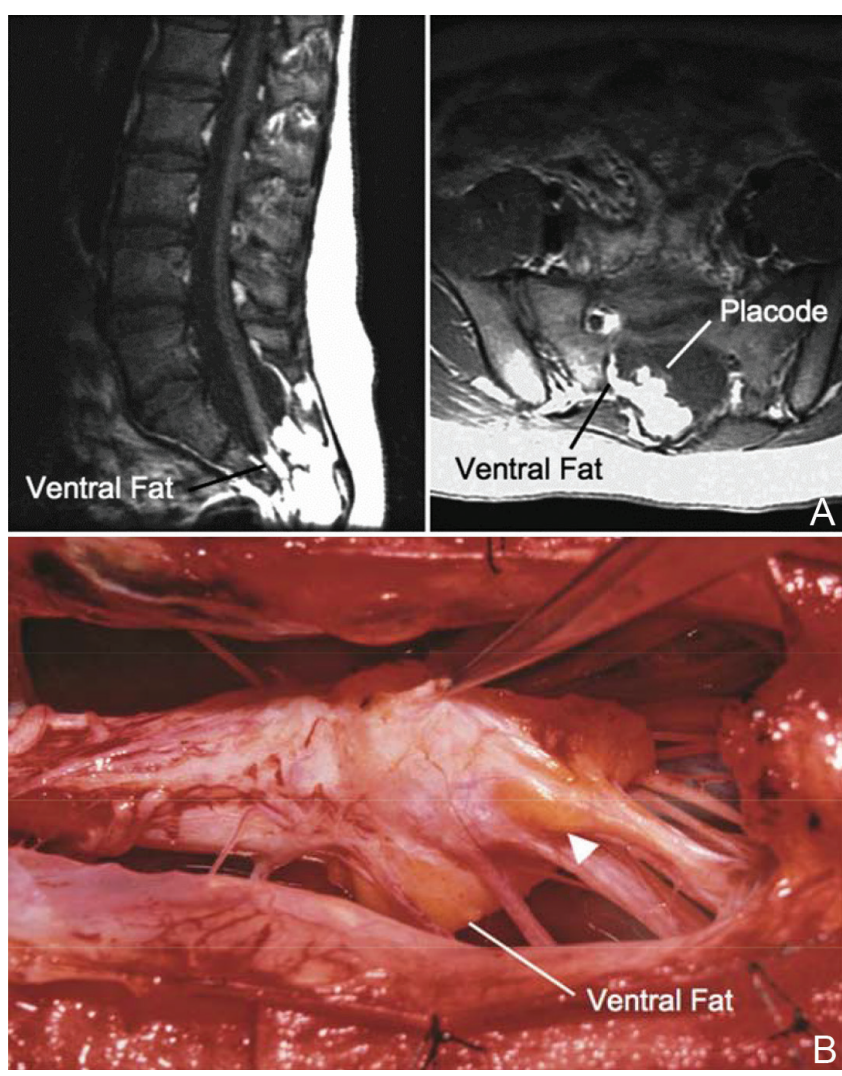

Fig. 4 Chaotic lipoma. A: Left: Sagittal magnetic resonance imaging shows ventral as well as dorsal fat in relation to the neural placode. Note sacral agenesis with only two visible sacral segments. Right: Axial image shows ventral fat and extremely irregular lipoma-fat interface. B: Intraoperative picture showing fat ventral to placode and on one of the sacral roots (arrowhead). Note absence of discrete fusion line. 


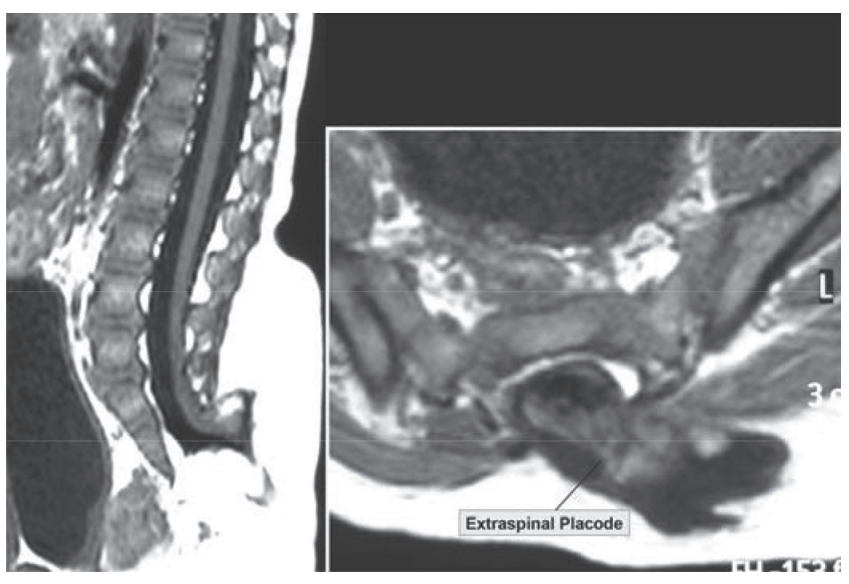

Fig. 5 Transitional lipoma with extraspinal extension ("lipomyelomeningocele"), with the lipoma, CSF sac, and part of the neural placode extending out of the spinal canal through a dorsal defect.

choose to classify this type as either a transitional or a dorsal lipoma with a descriptive qualifier of "extraspinal extension."

\section{Surgically Relevant Embryology}

An understanding of the embryogenesis of lipomas is helpful in appreciating the surgical nuances.

\section{Embryogenesis of dorsal and transitional lipomas}

In the embryo, a progressive disparity exists between the spinal cord and vertebral column as a result of the faster growth rate of the latter. ${ }^{8-11)}$ Even after accounting for partial dissolution of the distal neural tube during secondary neurulation, the caudal end of the cord still ascends from opposite the coccyx in the 30-mm human embryo to a much higher level at birth. ${ }^{10-13)}$ Proper ascent of the cord requires a well-formed neural tube and a smooth pia-arachnoid covering. If during early development a dorsal defect exists in the dura (duraschisis) and neural tube (myeloschisis), mesodermal elements from the surrounding mesenchyme will enter the dural sac and form attachment with the sliding neural tube in the form of a fibrofatty stalk, resulting in its entrapment. This theory features a fundamental defect in neural tube closure during primary neurulation (secondary neurulation does not involve dorsal neural fold closure), and thus applies only to the dorsal and transitional lipomas (see below). It is compatible with the observation that these two types of lipomas are always associated with neural arch defects.

In normal development, separation of the cutaneous ectoderm and neuroectoderm (disjunction) does not occur until after dorsal neural folds fusion. The
Embryogenesis of Dorsal Lipoma

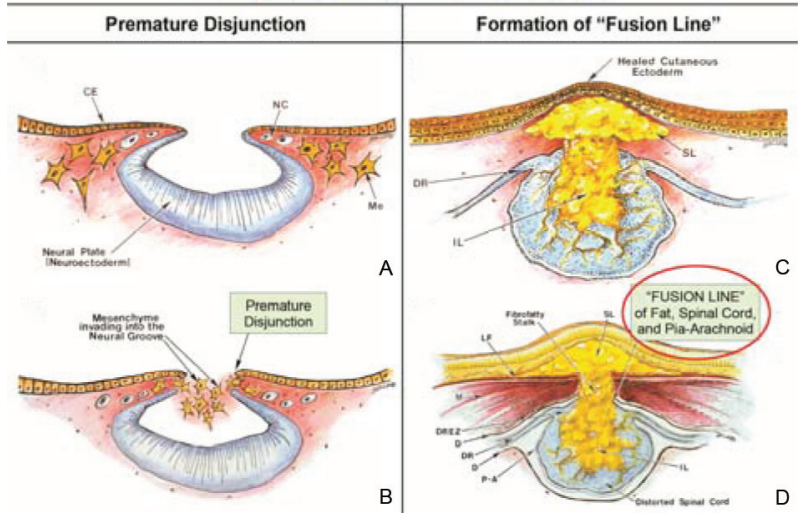

Fig. 6 Embryogenesis of dorsal lipoma, a pure primary neurulation defect. A, B: Premature disjunction before complete closure of neural plates allows migration of mesenchymal cells into neural groove to be in contact with the ependymal surface. C, D: Formation of fusion line between lipoma, cord, and pia-arachnoid. DREZ and dorsal root are always lateral to the fusion line and thus not entangled in fat. CE: cutaneous ectoderm, D: dura, DR: dorsal root, DREZ: dorsal root entry zone, IL: intramedullary lipoma, LF: lumbodorsal fascia M: muscle, Me: mesenchyme, NC: neural crest, P-A: piaarachnoid, SL: subcutaneous lipoma.

surrounding mesenchyme is thus always shielded from contact with the dorsal (ependymal) surface of the neural plate. The embryologic error leading to the mesodermal invasion of the neural tube probably lies in premature disjunction between the cutaneous and neural ectoderms; ${ }^{14,15}$ i.e., the separation of one from the other occurs before the converging neural folds fuse with each other. This allows the paraxial mesenchyme to roll over the still gaping neural folds and enter the central canal. Once contact between mesenchyme and ependymal neuroectoderm is made, further closure of the neural tube is prevented and a segmental dorsal myeloschisis is created (Fig. 6A, B). Alternatively, the fault may lie in a delay in neural folds fusion secondary to an insufficiency of the paraxial mesoderm in impelling their dorsal convergence, ${ }^{16-22)}$ so that ectodermal disjunction again precedes neural folds fusion. Finally, faulty fusion of the neural folds due to metabolic disturbance of the cell membrane-bound glycosaminoglycans, which are vital to cell-cell recognition and adhesion, ${ }^{23-26)}$ could likewise reverse the temporal relationship between disjunction and neural folds fusion.

Experimental studies show that the pluripotential mesenchyme forms derivatives according to the inductive properties of the adjacent neuroectoderm (Fig. 6C). ${ }^{27,28)}$ The ependymal side of the neural tube induces mesenchyme to form fat, muscles, collagen, 
and occasionally bone and cartilage, whereas the outer surface of the neural tube induces the formation of meninges. ${ }^{29)}$ However, no dura can now form over the dorsal opened portion of the neural tube, and the dural defect neatly surrounds the evolving lipomatous stalk, which tethers the neural tube to the subcutaneous adiposity. In like manner, deficiencies in the overlying myofascial layers (from myotomal mesoderm) and neural arches (from scleromesoderm) also neatly surround the lipomatous stalk (Fig. 6D).

Within the neural tube, the intramedullary fat and muscles fuse with the developing alar and basal plates. Since the dorsal root ganglions develop from neural crest cells at the outer aspect of the neural fold lateral to the site of failed fusion, the dorsal nerve roots grow outward ventrolateral to, but never traverse, the lipomatous stalk. The DREZ must correspondingly lie very near, but always lateral to, the exact junctional boundary between lipoma and spinal cord. This boundary, called fusion line, is of tremendous surgical significance (Fig. 6D). ${ }^{2,30,31)}$ Meanwhile, the cutaneous ectoderm, long detached from the neuroectoderm, heals over in the dorsal midline to form wholesome skin over the subcutaneous lipoma.

The genesis of dorsal lipoma perfectly exemplifies mistimed disjunction during primary neurulation. Its fibrofatty stalk always involves cord segments above the conus, which mainly forms from secondary neurulation. Furthermore, failure of primary neural tube closure appears to be segmental, normal closure takes place "business-as-usual" immediately following the abnormal event. This "square pulse" nature is illustrated by the fact that the sharp fusion line between fat, spinal cord, and pia-arachnoid can be neatly traced circumferentially round the lipomatous stalk (Fig. 2). ${ }^{2,30-32)}$ Dorsal lipomas therefore result from segmental closure abnormality involving only primary neurulation. They are found in less than $15 \%$ of spinal cord lipomas in our series. ${ }^{1-3)}$

In transitional lipoma, the myeloschisis involves much more than an isolated segment of the primary neural tube. Even though its rostral part resembles the dorsal lipoma, the involvement of the whole of the caudal spinal cord means that not only primary but also secondary neurulation have been profoundly disturbed by the mesodermal invasion. This is supported by the observations that in many transitional lipomas the filum is incorporated into the distal fat, and within the lipomas are often spaces resembling the vacuoles found within the secondary neural tube during its cavitary stage of development. Also, while the rostral part of the transitional lipoma is always dorsal and aptly reflects premature disjunction of primary neurulation, the distal part involves the core of the conus, a situation compatible with misguided mesenchymal inclusion during the much less orderly events of secondary neurulation. Intramedullary mesenchyme may migrate within the neural tube after invasion and travel caudally across the boundary from the primary to the secondary neural canal, since the two neural canals are in continuity. ${ }^{33)}$ In fact, the hypothesis that the rostral part of the transitional lipoma arises from aberrant primary neurulation (involving only the dorsal cord) and the caudal part arises from abnormal condensation of the secondary neural cord (affecting the deeper central core of the conus) furnishes at least one explanation for the dorsoventral obliquity of the lipoma-cord interface. In spite of the larger field of involvement of the neural tube, the lipoma-cord interface remains relatively distinct in most transitional lipomas.

\section{Embryogenesis of chaotic lipomas}

Chaotic lipomas do not quite fit into either the dorsal or transitional schema. They often do not have a distinct dorsal part with the symmetry of a dorsal lipoma, and the lipoma-cord interface is irregular and ill-defined, with fat running through the neural placode to the ventral side in large and unruly measures. Even in the context of the less orderly transitional lipoma, the interplay between lipoma and cord in this type of lesion seems to be in constant chaos.

This degree of anatomical unpredictability in chaotic lipoma and its strong association with caudal agenesis $\left(82 \%\right.$ in our series $\left.{ }^{2)}\right)$ suggest that the embryogenetic error occurs during the early stage of secondary neurulation as part of the general failure of the caudal cell mass (Fig. 7). ${ }^{34,35)}$ Secondary neurulation comprises three distinct stages: (1) condensation of neural material from the caudal cell mass to form the solid medullary cord; (2) intrachordal cavitation of the medullary $\operatorname{cord}^{33,35,36)}$ and its integration with the primary neural tube; and (3) partial degeneration of the cavitary medullary cord through massive apoptosis to result in the thin filum terminale. ${ }^{10,33)}$ It is possible that formation of the chaotic lipoma involves the entanglement of lipogenic mesenchymal stem cells with cells from the caudal cell mass during aberrant condensation of the medullary cord, forming an inseparable mixture of neural tissue and fat, with nerve roots projecting out haphazardly. ${ }^{2)}$

\section{Intraoperative Electrophysiological Monitoring}

Intraoperative electrophysiological monitoring is sine qua non in lipoma surgery..$^{2,3,37-39)}$ Elaborate 


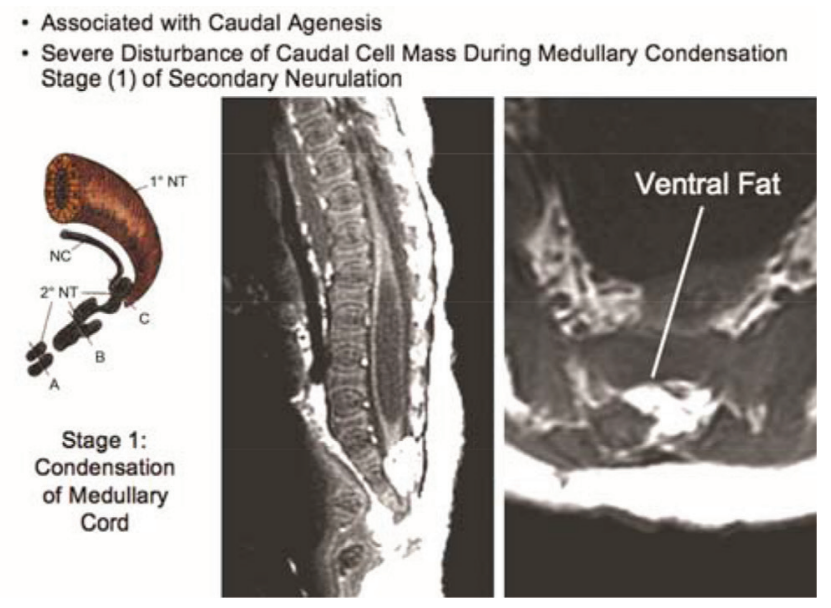

Fig. 7 Embryogenesis of chaotic lipomas. Left: Basic error probably occurs with inclusion of abnormal lipogenic mesenchymal cells into caudal cord during condensation stage (Stage 1) of secondary neurulation, with formation of the medullary cord, thereby generating fat tissue through the substance of the mature neural placode. Middle and right show dorsal and ventral fat and associated sacral agenesis. $1^{\circ} \mathrm{NT}$ : primary neural tube, $2^{\circ}$ NT: secondary neural tube, NC: notochord.

electromyographic preparations are made to capture triggered motor responses from the relevant lumbosacral nerve roots. Standard electromyographic needles are inserted into the rectus femorus (L4), anterior tibialis (L4-L5), gastrocnemius (S1), and abductor hallucis (S2). Smaller-gauge (No. 27) electromyographic needles are inserted obliquely into the external anal sphincter through the anal verge on each side, which is insulated from the other side with a plug of dry muslin gauze so that sphincter contractions from one side can be unequivocally distinguished from each other. ${ }^{37)}$ All stimulations and recordings are done with the Cadwell Cascade Intraoperative Monitoring System (Cadwell Lab. Inc., Kennewick, Washington, USA) using the Cascade Software Version 2.5.

Needle or pad stimulating electrodes are placed subcutaneously along the shaft of the penis in males and between the periclitoral skin and the labia minora in females. Stimulation of the sensory domain of the pudendal nerve via these electrodes generates an "electric" bulbocavernosus reflex (BCR), which is a form of $\mathrm{H}$ reflex for the conus useful in monitoring the integrity of the central connections of the sacral motor centre apart from motor root mapping. ${ }^{37,39)}$

Standard stimulating electrodes are placed near the posterior tibial nerve behind the medial maleolus and near the common peroneal nerve at the fibular neck to enable somatosensory evoked potential monitoring for the spinal cord segments above S2. ${ }^{37}$

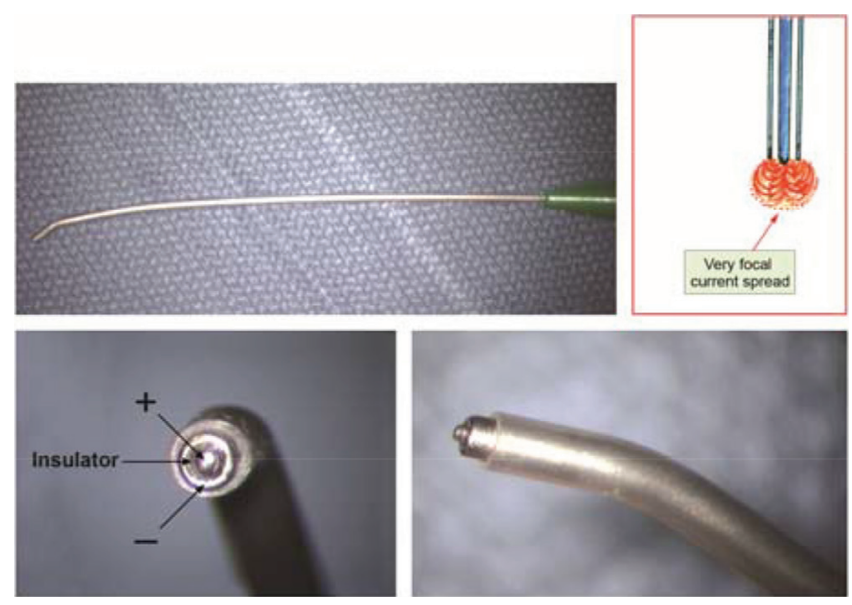

Fig. 8 Concentric coaxial bipolar microprobe stimulator in which the concentric cathode and anode are separated by a coaxial insulator. Tip diameter is approximately $1.75 \mathrm{~mm}$. The microprobe delivers very focal current spread (Inset).

All motor root and direct spinal cord stimulations are done with the concentric coaxial bipolar microprobe electrode with an end-plate diameter of $1.75 \mathrm{~mm}$ (Medtronic Xomed Inc., Jacksonville, Florida, USA) (Fig. 8). The concentric configuration and small size of the anode-cathode complex allow extremely focused current delivery to a very small target volume, thus making the electrode ideal for fine discrimination of small and crowded electroresponsive units. ${ }^{2}$ Stimulating currents from $0.3 \mathrm{~mA}$ to $3.0 \mathrm{~mA}$ are used depending on target impedence. Most functional motor roots will respond to currents of $0.3 \mathrm{~mA}$ to $1.0 \mathrm{~mA}$, though occasionally requiring $1.5 \mathrm{~mA}$ if the roots are partially fibrotic. Direct spinal cord stimulation will require a current of $2.0 \mathrm{~mA}$ or higher; both sides will be recruited if the probe is placed on the midline. The stimulation frequency is usually set at 10 per second. This permits spontaneous random firing due to nerve irritation from surgical manipulation to be distinguishable from the rhythmic evoked contractions.

To elicit transcortical motor evoked potentials (TcMEPs) from the external anal sphincter, the stimulation parameters are the same for the lower limbs: a trend of 8, each with a duration of $75 \mu \mathrm{sec}$ and intensity of 100-300 volts. The inhalation anesthetic concentration, e.g., of sevoflurane, is usually adjusted to no higher than 0.8 Mac to improve maximum yield. Given that the BCR can be variable or even unobtainable in infants, and it is also exquisitely sensitive to inhalation anesthesia, the TcMEP is an important adjunct for monitoring the corticospinal input to the sacral motor center. 


\section{Surgical Technique of Total/New Total Lipoma Resection}

The description below is based on a case of an unusually large and complex dorsal lipoma that illustrates most of the key technical points of total resection applicable to dorsal and transitional lipomas. The case is that of a 12-month-old girl who was born without neurological deficits but had diagnostic magnetic resonance imaging (MRI) because of a subcutaneous lump and gluteal cleft deviation. At 12 months of age she developed left leg weakness and repeat MRI showed massive enlargement of the lipoma (Fig. 9).

\section{Step 1: Exposure and Dealing with the Extraspinal} Lipoma Stalk

Accurate localization of the skin incision is important to ensure adequate bony exposure, which should span at least one level rostral to the rostral-most part of the lipoma-cord interface, and caudally at least $1 \mathrm{~cm}$ distal to the end of the conus. Two modified metal paper clips are taped to the back at the estimated upper and lower extent of the laminectomy for a lateral radiograph, which is then matched up with the sagittal MRI to finalize the skin incision (Fig. 10).

The skin and soft tissue dissection goes straight through the subcutaneous lipoma. Removing the latter will leave behind a large subdermal space into which cerebrospinal fluid (CSF) could collect under tension and consequently hinder wound healing. Frequently, a discrete lipoma stalk connects the intraspinal to the subcutaneous fat; it is sharply

\section{Dorsal Lipoma: 1 Year Old, Slightly Weaker Left Leg}
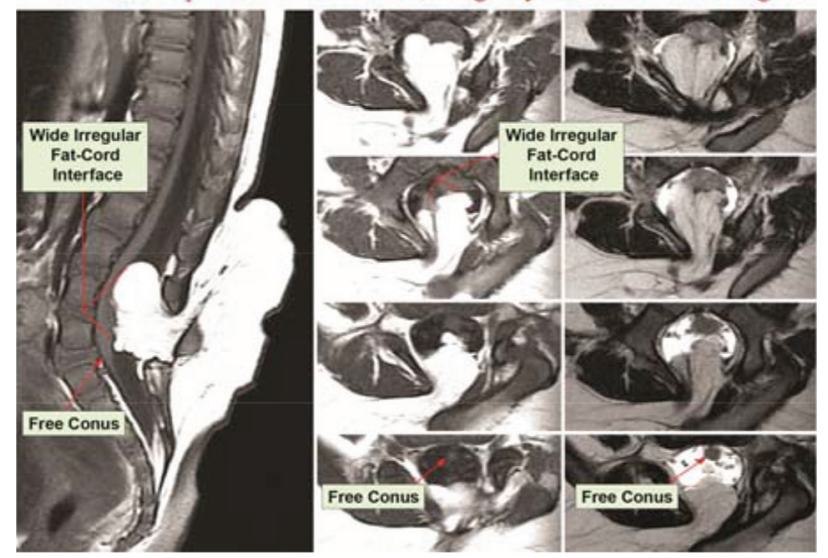

Fig. 9 Magnetic resonance imaging of a 12-month-old girl with left leg weakness shows a large dorsal lipoma. The sagittal image shows a long stretch of lipoma-cord interface, but the conus is clearly free of fat. The axial images reveal the irregular nature of the lipoma-cord interface, but the neural placode is not excessively tilted to one side. isolated and traced through a defect in the lumbodorsal fascia (Fig. 11). This stalk is attached to the spinal cord and cannot be tugged on during fascial dissection.

The upper extent of the bony exposure should include one level above the rostral end of the lipoma to ensure good visual orientation. Wide laminectomy is absolutely essential to afford full access to the lateral edges of the dural sac (see below). Visualization of the normal dura rostral to any lipoma gives a depth perspective as to how far neural tissue and CSF sac might have extruded beyond the plane of the dura. The heavy bulk of the extradural lipoma stalk could then be safely lopped off to give room for intradural manipulation and to lighten the tug on the conus (Fig. 11).
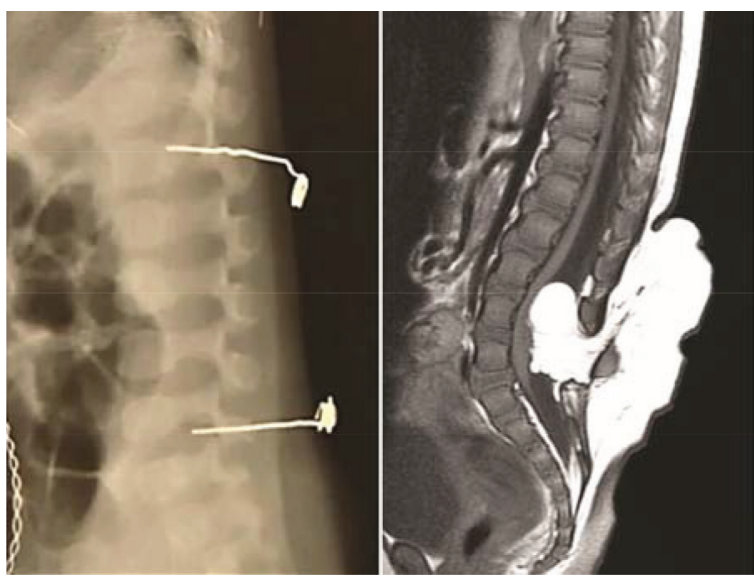

Fig. 10 Lateral radiograph showing the localizing markers is matched to the sagittal magnetic resonance imaging to finalize the top and bottom extent of the exposure.

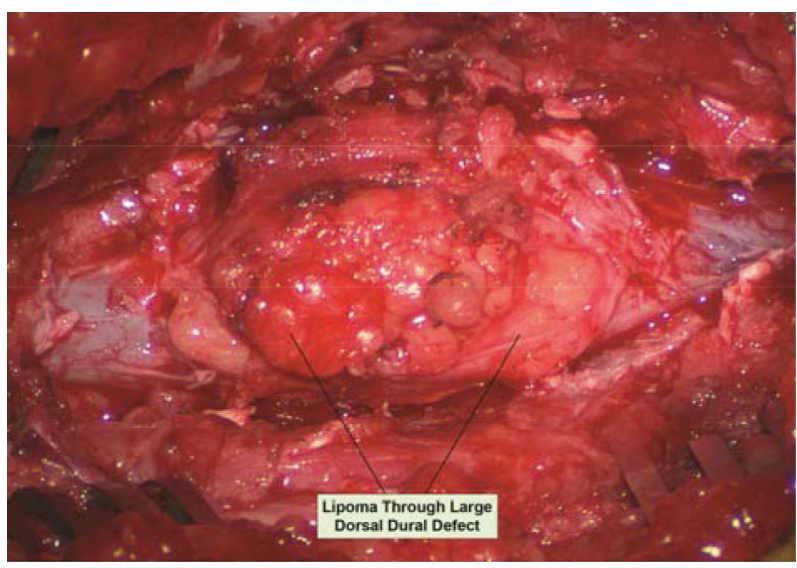

Fig. 11 Large extradural portion of the lipoma before dural opening. Note huge dural defect through which the extradural lipoma extends. Rostral exposure is to the right. Note the essential wide bony exposure. 


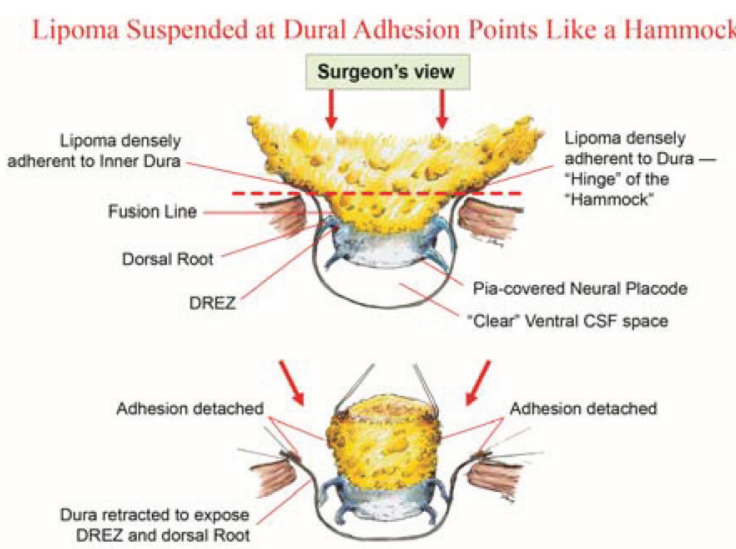

Fig. 12 Schema depicting the relationship among the lipoma, neural placode, nerve roots, and dural sac in an axial slice. Upper: The lipoma-cord assembly is suspended at the dural edges at far lateral adhesion points like a hammock against side hinges. The dotted transverse line that joins the two side hinges divides the assembly into a dorsal disorderly, fibrofatty half that completely blocks the surgeon's view to a much more orderly ventral half, containing the important anatomical landmarks of fusion line, DREZ, dorsal roots, fat-free ventral placode, and pristine ventral CSF space. Lower: After detaching the far lateral adhesion points (the hinges) by careful "crotch dissection," and folding-in the fatty mass, the ventral anatomical landmarks can now be visualized.

\section{Step 2: Detachment of Lipoma from Dura: Unhinging the Hammock}

The dura is opened in the midline about $1 \mathrm{~cm}$ rostral to the rostral extreme of the lipoma. The dural edge on each side is tautly and widely retracted with sutures. This is a crucial maneuver because full lateral exposure of the intradural span, made possible by the generous bone removal, reveals the "crotch" where the far lateral fringe of the lipoma attaches to the inner surface of the dura. To understand the importance of the "crotch," one must recognize that in each successive axial slice, the lipoma is roughly divided by a transverse line joining the points of the far lateral fat-dura attachment on each side, where the lipoma-cord assembly is in effect suspended from the inner dura like a hammock against two lateral hinges over an uncluttered ventral CSF pool. Dorsal to this transverse line is the visible but disorderly, massive, and unrevealing fat, and ventral to this line is the orderly fusion line, DREZ, dorsal nerve roots, neural placode, and ventral CSF space but all initially rendered invisible to the surgeon by the overhanging fat (Fig. 12, upper). Prior to further dissection, this suspension must be released so that the hammock of neural placode and nerve roots

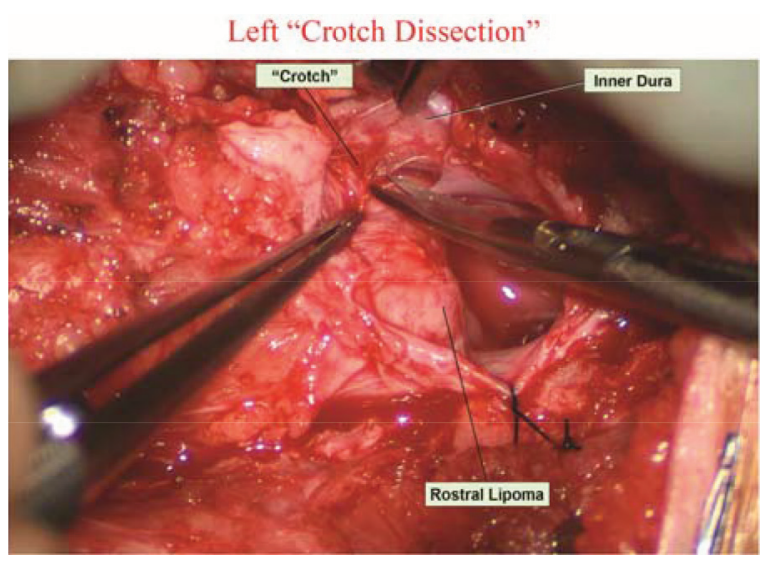

Fig. 13 Left crotch dissection in the large dorsal lipoma shown in Fig. 9. The lipoma is grasped firmly and pulled gently away from the adherent points on the inner dura, stretching the adhesion bands and thick arachnoid, creating the "crotch" and making the adhesions safe to be cut.

can be folded inward enough to be identified and preserved (Fig. 12, lower).

To perform "crotch dissection" and unhinge the hammock, the fat overhanging the fusion line is grasped and pulled medially under tension against the tagged dural edge whilst the dissecting scissors sharply divide the fat-dura attachment (Fig. 13). It is absolutely requisite to lean the round curve of the scissors firmly against the inner lining of the dura while cutting this attachment to avoid blindly injuring the nerve roots, which project from the cord slightly medial to the "crotch" and lie just deep to the fat. The hidden roots should spring into view wherever the detached fat is pulled back (Fig. 14A, B), and can be gently coaxed away from the dura by blunt dissection all the way to the exit foramina (Fig. 15). At the same time, the free CSF space ventral to the nerve roots, and the fat-free, pia-covered ventral surface of the neural placode, hitherto hidden by the overhang, now "pop" into view (Fig. 16). This laborious but indispensable step of "crotch dissection" is carried all the way caudally until all the functioning nerve roots are identified and the entire neural placode, with a profusion of lipoma still attached, is completely unsuspended from the dura and has literally "fallen" to the basin of the dural trough (Fig. 17).

Identifying the "crotch" is not always a simple affair. When the dura is so attenuated that it clings to the side of the lipoma and the spinal cord like pia mater, as in the left side of the case here, the surgeon can mistakenly stray into the extradural space and lose all perspective of anatomy. Dissecting into this space will encounter the extradural roots 

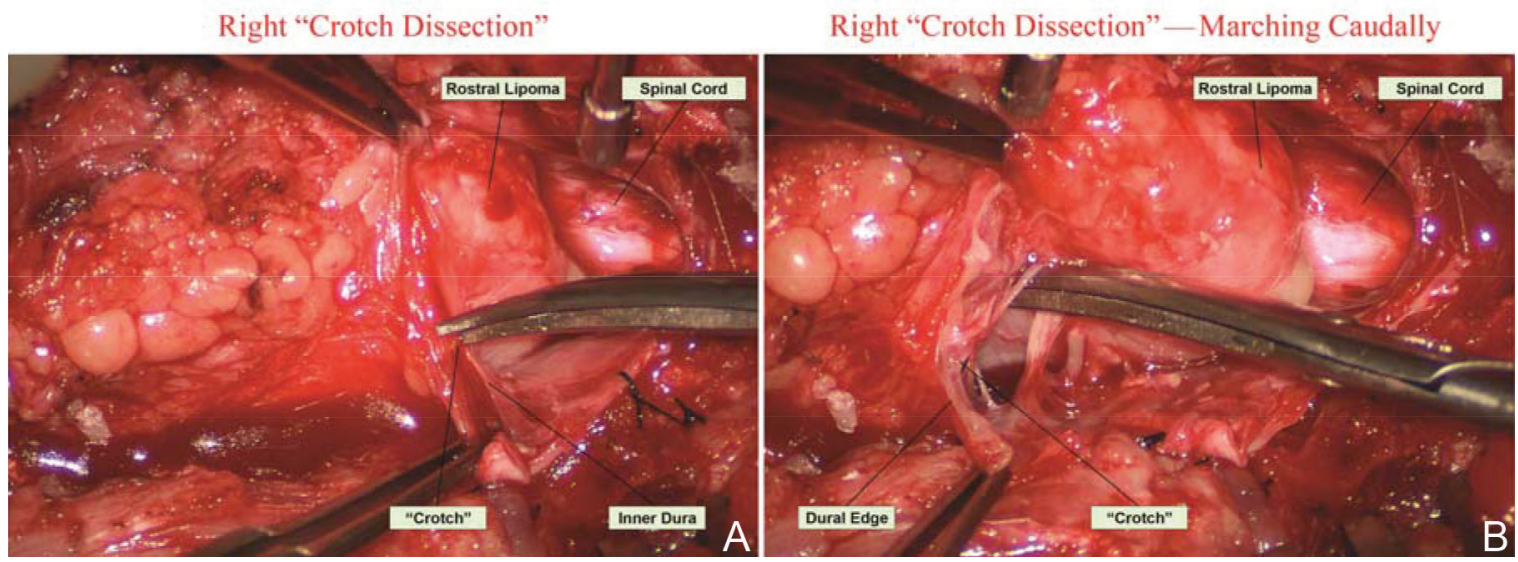

Fig. 14 Right crotch dissection. A: Cutting of the "crotch" on the right side as in Fig. 13. B: Lysing the crotch exposes the hidden nerve roots, the ventral neural placode, and the ventral free subarachnoid space.

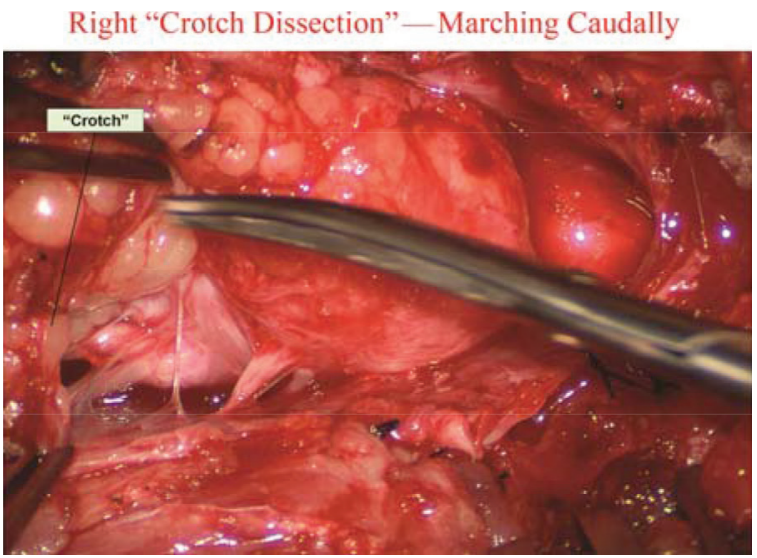

Fig. 15 Marching caudally with the right crotch dissection, the surgeon exposes the ventral surface of the placode, the free ventral subarachnoid space, and more nerve roots that can be traced all the way to their exit foramina.

enmeshed in fat, and render them at risk of being inadvertently cut. The best option if caught in this dilemma is to abandon further intervention on the left and retreat to open the crotch on the easier and safer right side. Progress here is smooth and brisk. After the surgeon dispatches with the lipomadura adhesions on the right side and resumes firm command of the ventral subarachnoid space on both sides, he can then trace along this space back to the left side and pick up the left crotch dissection in more propitious light (Fig. 18).

\section{Step 3: Lipoma Resection}

In preparation for lipoma resection, the fusion line is identified where pia, spinal cord, and lipoma join in a continuous furrowed border that travels from rostral to caudal outlining the entire attachment of

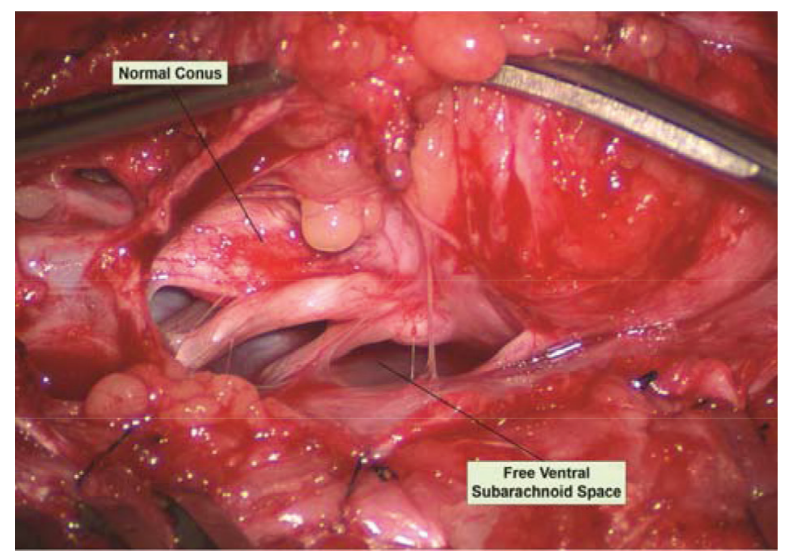

Fig. 16 After crotch dissection on the right side and complete detachment of the lipoma (hammock) from the right inner dural lining, the fat-free ventral subarachnoid space, caudal nerve roots, and conus are well seen.

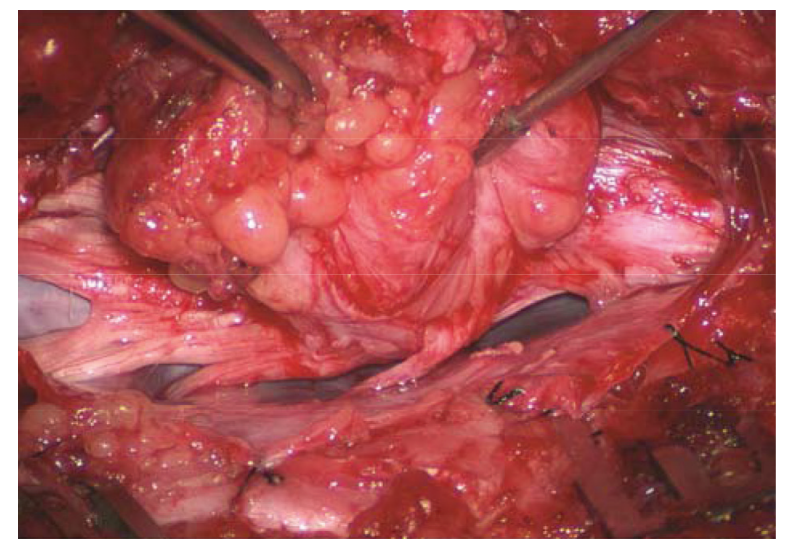

Fig. 17 Crotch dissection is complete on both sides. The entire right array of nerve roots are seen, but not the DREZ or the fusion line, which are covered by the lateral overhang of the large lipoma. The entire hammock is now unsuspended from the dura. 


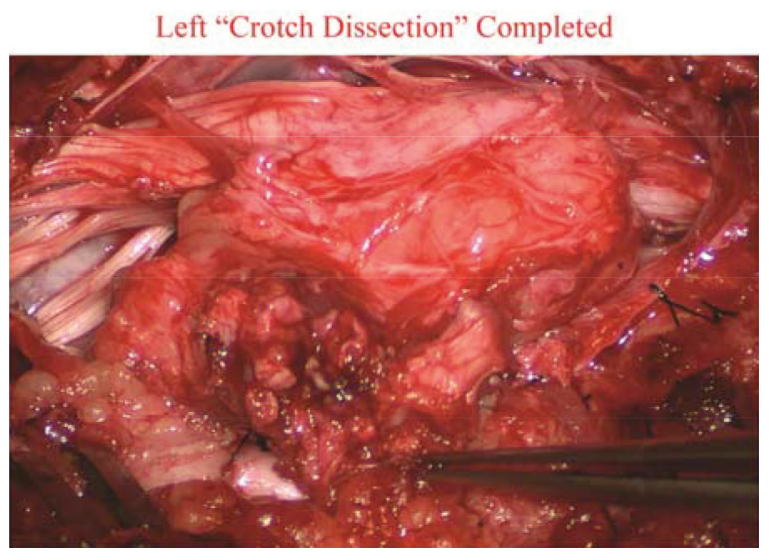

Fig. 18 Complete crotch dissection on the left side, showing left nerve roots and ventral placode surface. Anatomy here is not as distinct and normal as on the right.

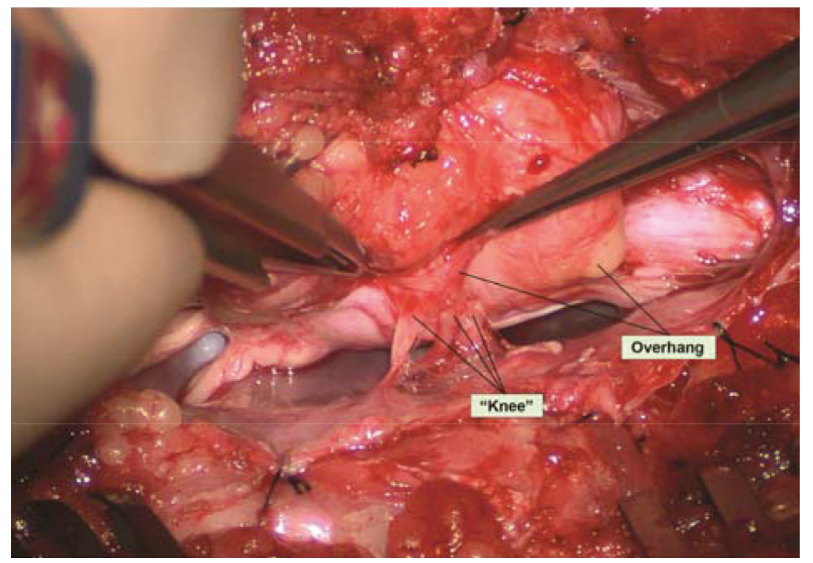

Fig. 19 The lateral overhang of the lipoma is well appreciated. Only the "knees" of the dorsal roots are seen. The "thighs," or the most proximal portions of the roots, are hidden by and adherent to the overhanging fat.

the lipoma stalk to the cord. In a dorsal lipoma, the fusion line forms a neat complete oval or circle from side to side, usually upon a leveled horizontal plane, often bilaterally symmetrical, and always sparing the conus below (Fig. 17). In a transitional lipoma, the rostral fusion line starts distinctly enough but then edges ventrally towards the tip of the conus and tends to wander laterally and asymmetrically, often becomes sheltered by the overhanging fat, and never meets its mate from the other side at the caudal end (Fig. 3A, B). For a large lipoma of either type, as in the monstrous dorsal lipoma shown in Fig. 9, festoons of overhanging fat cover up the fusion line and obscure the upper surface of the emergent dorsal roots (the "thighs" of the roots) to give the false impression that they course through and not underneath the lipoma

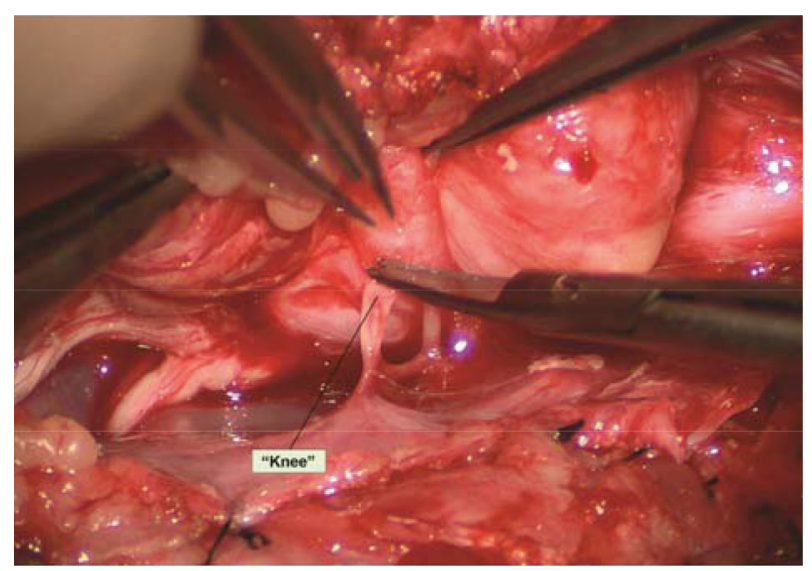

Fig. 20 "Knee dissection": the adhesions covering the "thigh" of the dorsal roots are sharply taken down.

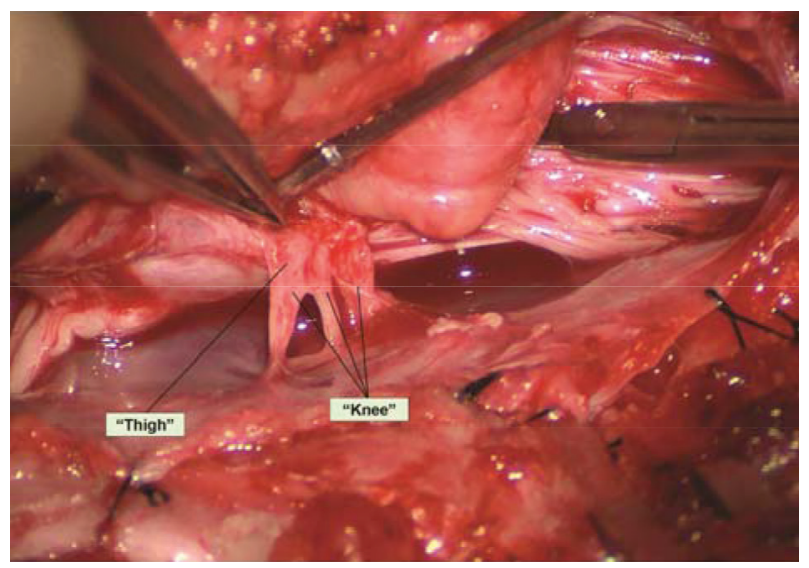

Fig. 21 After ridding the adhesions, the proximal "thigh" portions of the dorsal roots are exposed.

(Fig. 19). With sharp dissection of the adhesions on the "thighs," the overhang can be readily teased and lifted off the "knees" of the roots (Fig. 20) to allow them to be re-traced under the verandah of fat into the true DREZ (Fig. 21). The true fusion line can now be picked up along the furrow (Fig. 22). I call this maneuver "knee dissection."

After the fusion line on both sides are completely and confidently defined (Fig. 23), actual resection of the lipoma can commence. Resection always begins at the rostral end where the anatomical relationships between fat, DREZ, and nerve roots are clearly decipherable (Fig. 24). Sharp dissection with micro-scissors is used to locate a thin but distinct silvery white plane between fat and cord at the demi-lune of the rostral fusion line (Fig. 25). The white plane is a filmy netting of relatively compact collagen fibers. It can be extremely contorted and asymmetrical in the main bulk of the lipoma, but in 


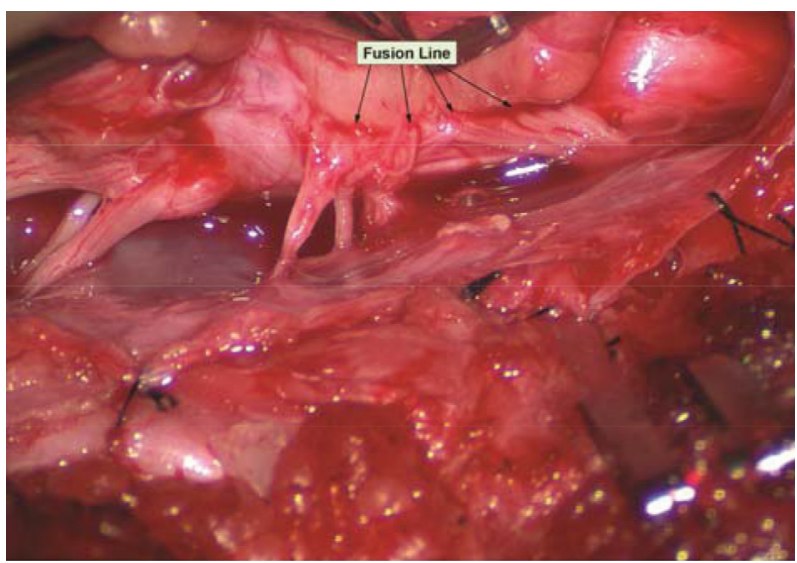

Fig. 22 The "true" fusion line on the right is revealed. Resection of lipoma on the lateral margin of the placode is now made safe.

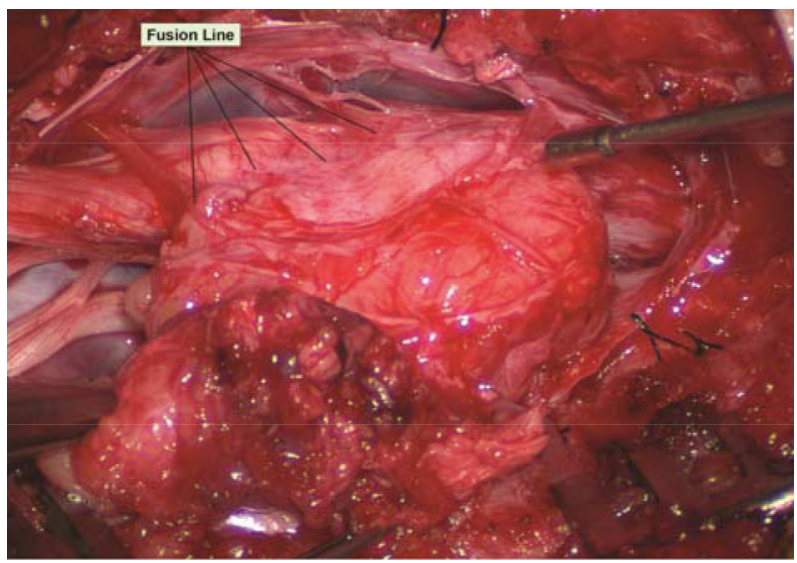

Fig. 23 The fusion line on the left is not as distinct as the right side and not on an even level.

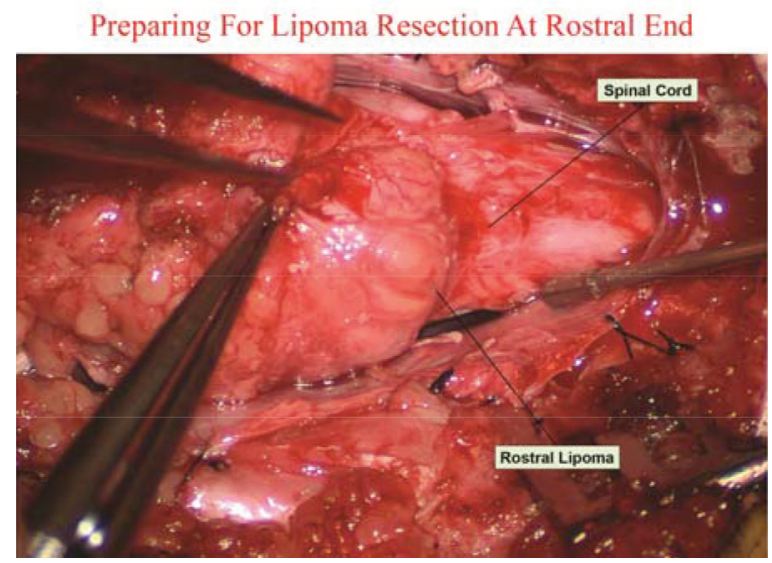

Fig. 24 Preparing for lipoma resection commencing on the rostral end of the fat where the lipoma-cord junction, the rostral dorsal roots, and beginning of the fusion lines are most distinct.
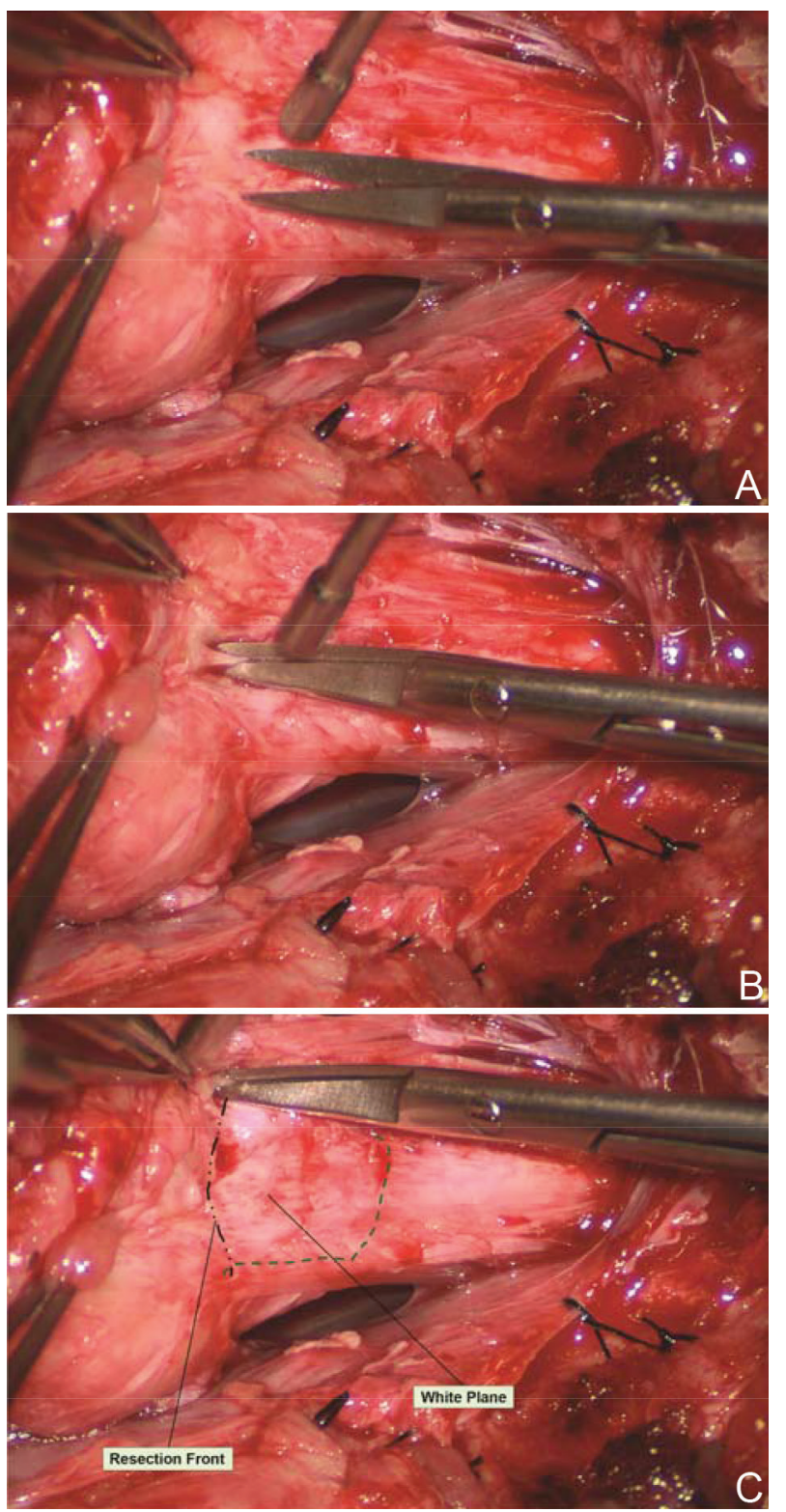

Fig. 25 Finding the white plane. A: Beginning resection at the rostral extremity of the lipoma. B: Boldly cutting sharply into the gritty fibrofatty base of the fat lump to locate the thin white plane. C: White plane located, which is a discrete though thin layer of whitish fibrous netting separating fat from spinal cord.

the upper extreme of the lesion, the white plane is manageably distinct (Fig. 26), and there is no better way than boldly cutting into the tongue of the rostral lipoma to find the glistening fibres beneath the first few globules of yellow fat. It takes some determination, for the initiate, to cut into this traditionally forbidden place, seemingly straight into the spinal cord, but with experience, this white plane can be found in every case (Fig. 27). Once the plane is located, one can follow it with sharp dissection 



Fig. 26 Resection of lipoma along the right fusion line. A: Cutting sharply on the white plane near the right fusion line. B: Note the pial fringe, carefully preserved for neurulation.

by feeling the grittiness through the micro-scissors and by spotting the glistening white stripes between yellow fat and pink spinal cord (Fig. 28). Frequently, the micro-scissors cannot handle the toughness of the fibrous layer, and the larger, sturdier Jamison Scissors have to be substituted. We strongly discourage using the $\mathrm{CO}_{2}$ laser to vaporize the fat because it chars the surface and thus blurs the safety net of the white plane. Its hands-off nature also negates the tactile feedback from the micro-scissors on which the surgeon depends to differentiate between clipping the slight grittiness of fibrous fat, the tougher white plane, and the formless softness of spinal cord. The Nd-YAG laser may produce slightly less blackening of the target surface but, lacking the contact feedback, is hardly better in navigating the dissection plane.

Bleeding on the white plane, which is only a hair's breadth from the spinal cord, must be handled delicately. The exact bleeding spot can be accurately and atraumatically located by intermittent gentle jets of irrigation through a 25 -gauge blunt needle affixed to a small hand squeezer that ophthalmic surgeons use in intraocular procedures. I regularly
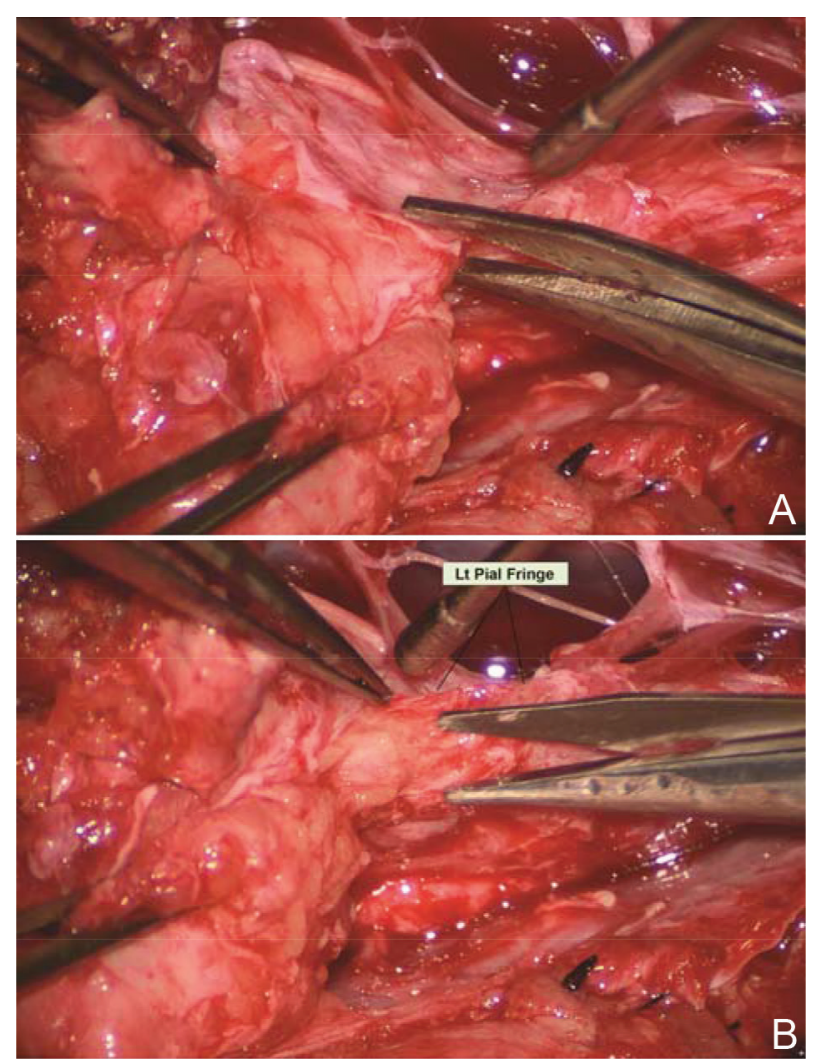

Fig. 27 Resection of lipoma along the left fusion line. A: Cutting lipoma from fusion line. B: Note white plane and left pial fringe.

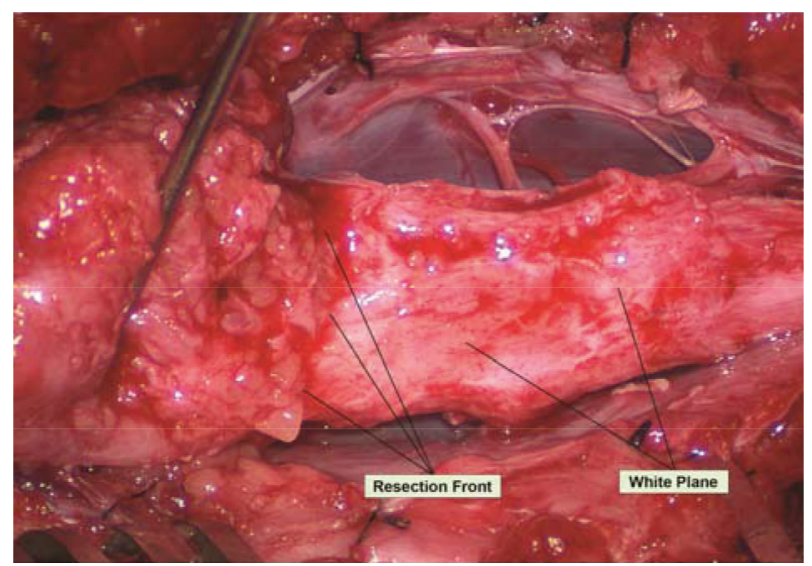

Fig. 28 The white plane, with the unresected portion of the lipoma on the left lifted up to show the resection front as a well-defined transverse line across the body of the neural placode.

use a No. 3 or No. 5 suction cannula with rounded tips on the resection front, but even these could cause inadvertent gouging on the soft neural placode whilst scrambling to control the bleeding. Once the bleeding source is precisely located, it can be deftly handled with the ultra-fine irrigating bipolar 
cautery (0.2 mm tips, Fig. 29) and a very low current setting. The cold irrigation militates against sticking but more importantly it dissipates heat rapidly from the cord. Minimal diathermy is used on the DREZ to avoid post-operative dysesthesia.

In removing lipoma along the fusion lines (Fig. 30), one must resist against too much "gouging" at the DREZ, but more importantly, an adequate pial fringe at the lateral resection front, just medial to the DREZ, must be preserved to provide a foothold for the pial sutures at neurulation (Figs. 26A, B, 27B). As long as all the activities are rendered medial to the fusion line, thus also medial to the DREZ and nerve roots, dissection along the white plane can be conducted safely all the way to the end with no damage to the cord or nerve roots. In most dorsal lipomas, this is a simple feat because the white plane is basically horizontal and flat, the two banks are symmetrical, and the caudal end well defined rostral

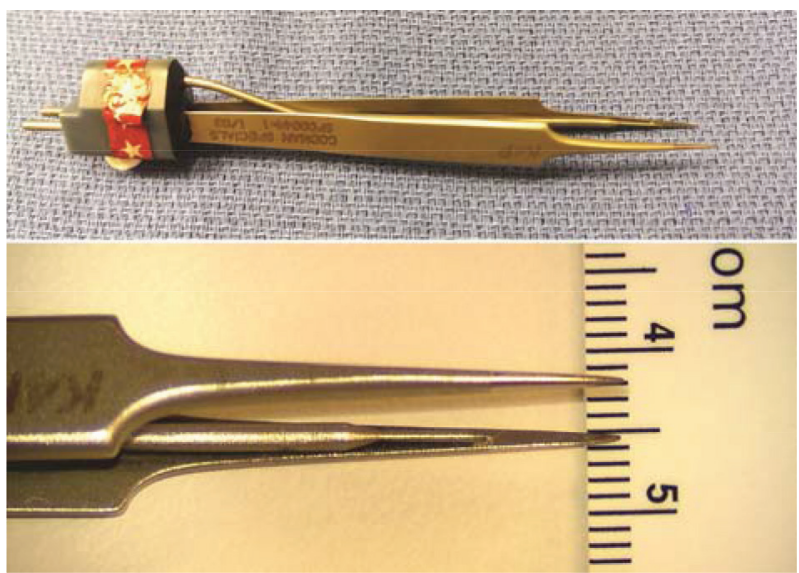

Fig. 29 Micro-irrigating bipolar cautery with super-fine tips measuring less than $0.2 \mathrm{~mm}$.

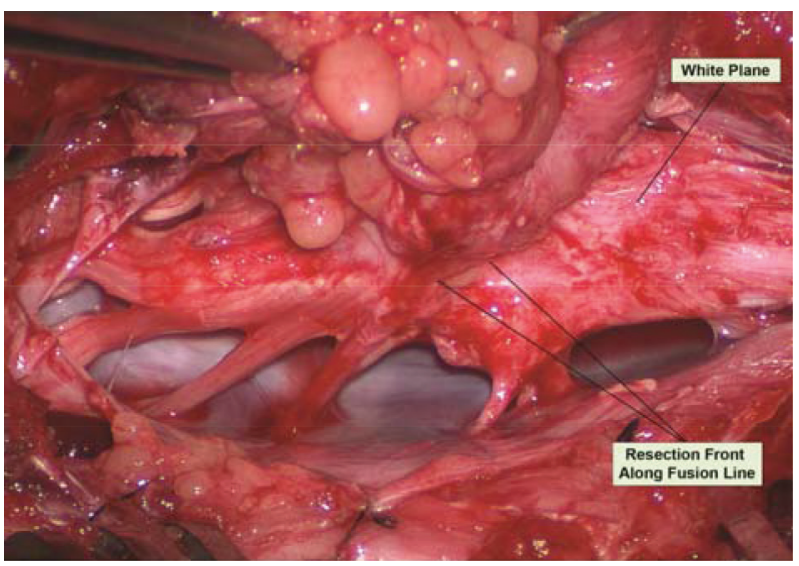

Fig. 30 Lateral white plane dissection along the right fusion line is almost complete. to the conus so that a completely circumscribed attack on the fat is possible from multiple angles (Fig. 31). In a particularly large and asymmetrical dorsal lipoma, navigating the white plane can be tricky because it tends to undulate with the uneven fusion line on the "bad" side, and one must be aware
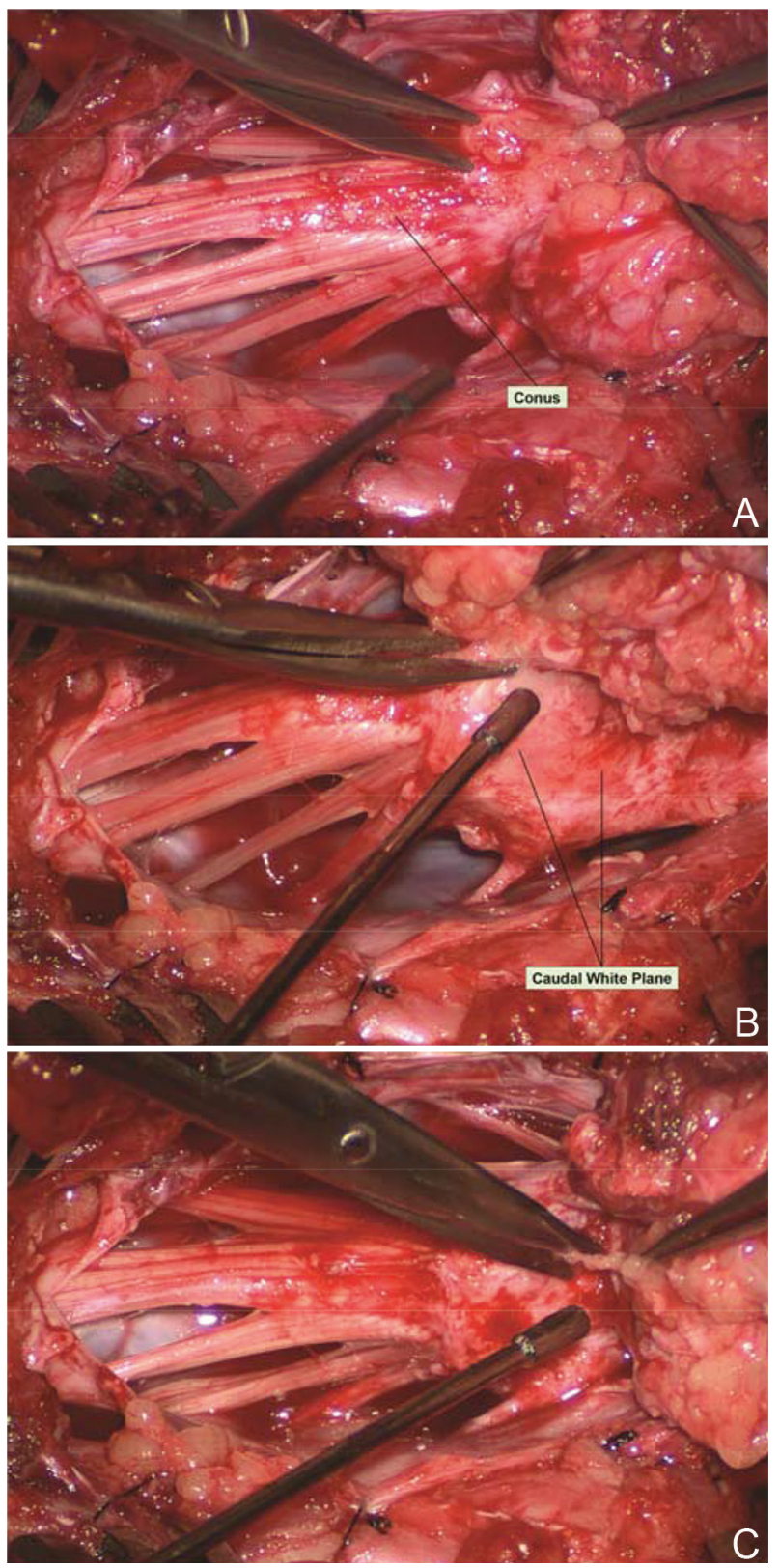

Fig. 31 Resection of the most caudal portion of the lipoma in the caudal to rostral direction, possible because this is a dorsal lipoma. The caudal white plane thus created will eventually merge with the proximal white plane resulting from the previous rostral-to-caudal dissection. A: Beginning white plane dissection from the conus side. B: More caudal white plane exposed. C: Last cut before completing white plane dissection. 
that the location of the DREZ may be at varying heights at different levels (Fig. 23). Locating this safe zone of dissection may require constant use of the concentric stimulating probe on the nerve roots.

In a large transitional lipoma, navigating the white plane is more difficult because it always slopes ventrally, often undulates, and one edge of it may be tilted so steeply that the corresponding DREZ and nerve roots are shifted ventrally and the placode so rotated that its ventral surface now faces the side of the spinal canal. Such a white plane is almost turned vertically "on edge," its orientation confusing unless one remembers the transverse line concept dividing one "clean" ventral hemisphere from the "messy" dorsal hemisphere. The white plane also sometimes seems never-ending in these lipomas and the caudal thecal sac is thronged with fat admixed with suspicious strands. This is when systematic stimulation and identification of the ventral nerve roots become invaluable in localizing the termination of the functional spinal cord. The $\mathrm{S}_{2}$ ventral root is readily identified when both the anal sphincter and the abductor hallucis contract using a low current of $0.3-1.0 \mathrm{~mA}$, and the next two sets of caudal rootlets with "pure" sphincter response are thus $\mathrm{S}_{3}$ and $\mathrm{S}_{4}$. As soon as these sphincter roots are found and preserved, the tissue distal to the last "live" pair can be considered non-essential and be cleanly cut across to consummate the final liberation of the placode (Fig. 32B). A good chunk of the now isolated distal fatty stump should be excised to prevent reconnection with the terminal placode.

In chaotic lipomas, electrophysiological determination of the functional extent of the neural placode may be the only way to achieve final untethering; the caudal fat-cord-fibrous jumble can only be sorted out functionally and not anatomically by direct stimulation of the placode and the projecting nerve roots. The handling of the white plane on the dorsal side of a chaotic lipoma is the same as with the other lipoma types, but the billows of fat on the ventral side of the placode should be left alone and its smooth pial surface left unviolated (Fig. 32). It is always the dorsal and never the ventral part (unless iatrogenically invaded) of the lipoma that actually tethers the spinal cord. ${ }^{2)}$

I generally prefer not to cut into the lipoma during resection for ease of handling because lacerated fat tends to be leaky and stringy and may confound the resection front on the white plane. Also, it is always more satisfying to grasp on to sturdy well-formed lipoma whilst detaching it from the cord than teasing on wet, drippy, whipped-up fat. However, I have encountered truly massive lipomas that completely obscure normal anatomy, of whose
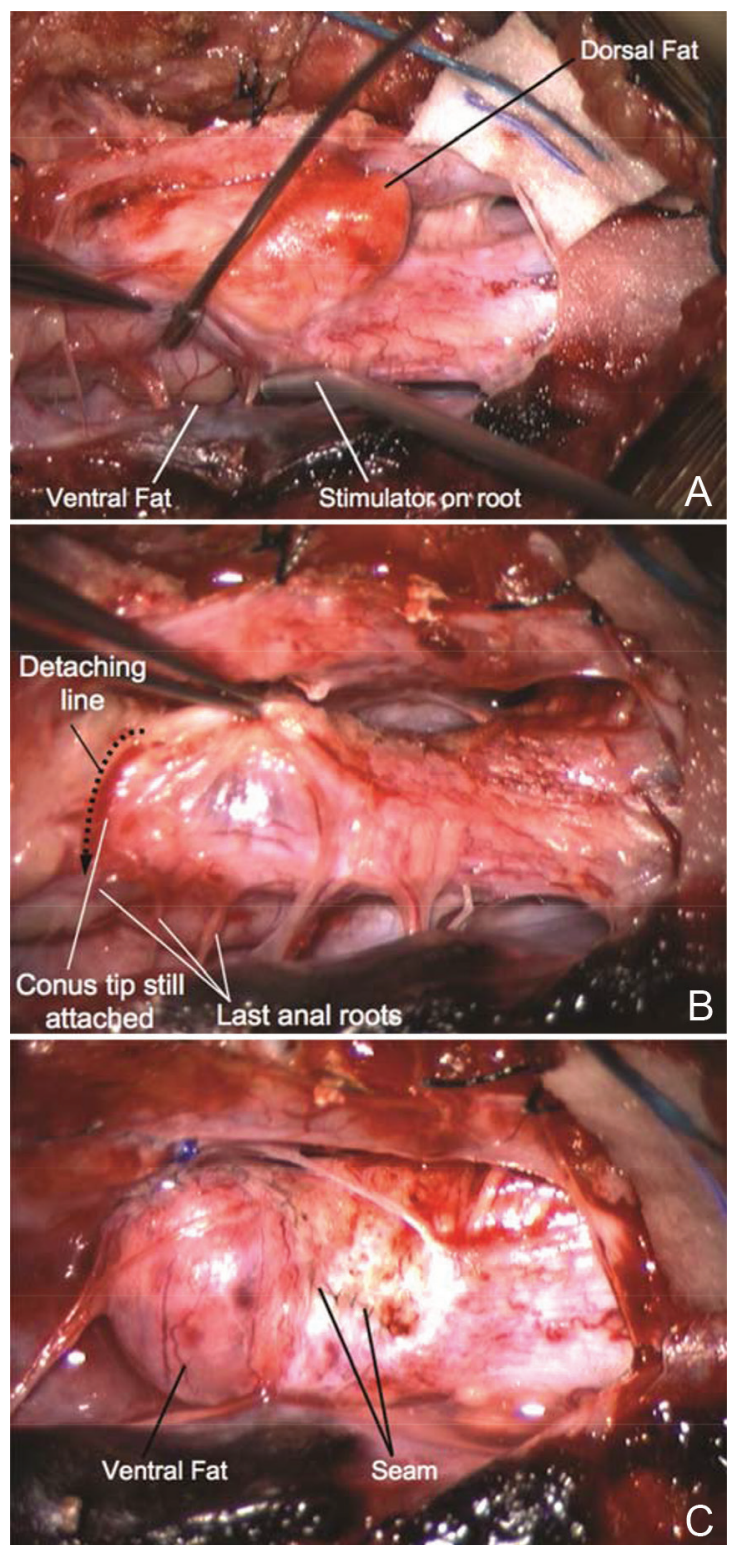

Fig. 32 Surgery for chaotic lipoma. A: Note ventral pia-covered fat medial to ventral nerve roots (being stimulated by concentric microprobe), and dorsal fat perched on the dorsal side of the placode. B: Terminal disconnection of neural placode from residual caudal lipoma stump after identification of three healthy pairs of anal sphincter motor roots. C: Caudal placode pulled up dorsally width-wise to be neurulated with the more proximal pial edge to form the seam, displaying the unviolated pia-covered ventral fat as a blunt stump.

central core had to be debulked with the ultrasonic aspirator before the sides could be collapsed inward to reveal the dorsal roots.

After complete resection of the lipoma (Fig. 33), it is good practice to systematically stimulate each set of nerve roots along the "naked" lipoma 


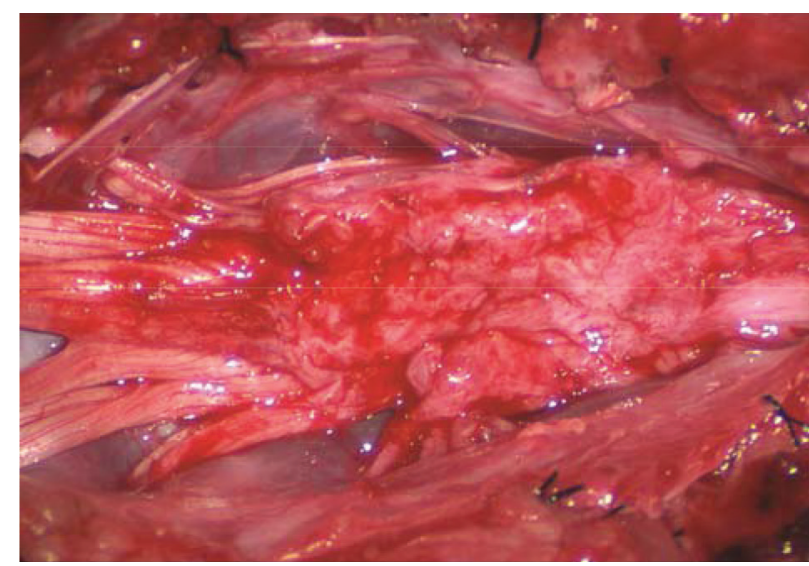

Fig. 33 Continuing from Fig. 31, the "naked" neural placode after complete resection of the dorsal lipoma.

bed to ensure all functions are preserved. The $S_{2}$ ventral root is readily identified when both the anal sphincter and the abductor hallucis contract using a low current, and the next two sets of "pure anal" caudal rootlets are $\mathrm{S}_{3}$ and $\mathrm{S}_{4}$.

Step 4: Pia-to-Pia Neurulation of the Neural Placode Thorough lipoma and scar resection impart the optimal bulk, texture, and maneuverability to the neural placode for tensionless pia-to-pia neurulation (Fig. 33). In turn, well-executed neurulation reduces the adhesive surface on the placode to a single seam, and, by transforming a broad, wafery, sticky sheet into a trim, sturdy pia-covered tube, duly minimizes the probability of its interacting with the dura.

Several technical hints and pitfalls deserve special mention. During the dorsal folding of the placode and side-to-side apposition of the pial edges, a substantial torque due to the natural tissue modulus of the placode may be encountered, ever tending to unfurl the undertaking. This can be temporarily counteracted by applying micro-Weck clips on the apposed pial fringes at short intervals along the seam (Fig. 34), whilst 8-0 nylon pia-to pia micro-sutures are being put in between the clips, always sewn in with the knots buried. After enough sutures are in place to withstand the torque, the Weck clips are successively removed.

Occasionally, strong tugging on the inbending folds of the placode is felt that prevents their free coaptation, exerted by adjacent clusters of "short" nerve roots (Fig. 35). These "short" and presumed to be "fibrotic" nerve roots have been reported by others as obstacles to thorough untethering of the cord and unjustly blamed for treatment failure. In reality, these normal functioning and perfectly pliant roots are tightly bound to the inner side wall

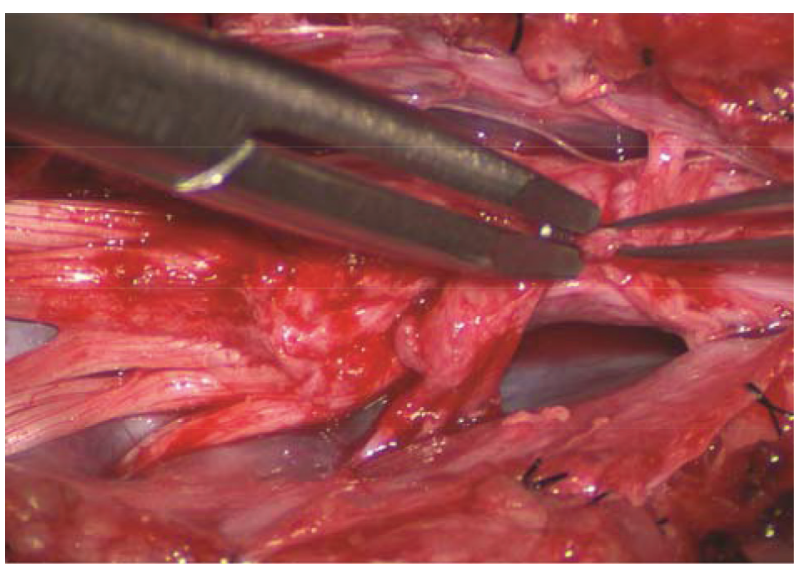

Fig. 34 Temporary small Weck clips are applied to the apposed pial fringes to absorb the tissue torque tending to unfurl the dorsal bending of the placode.

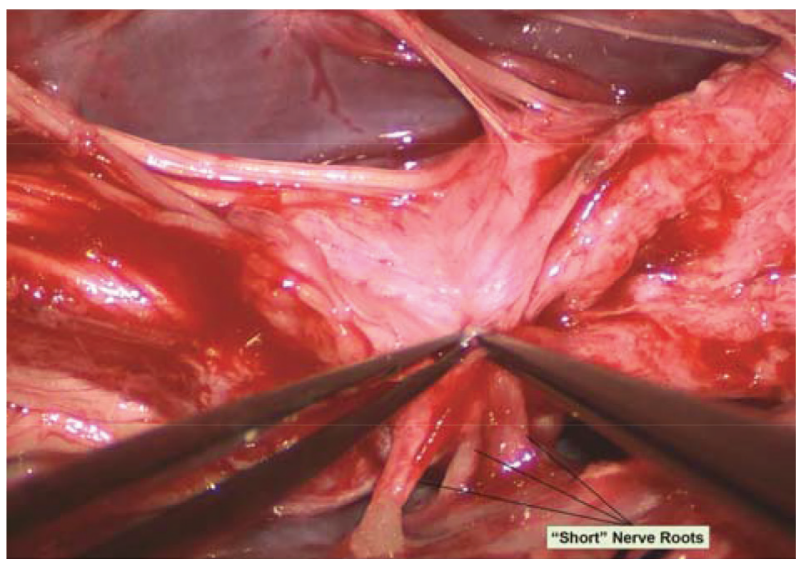

Fig. 35 On pulling the pial fringes together to check the ease of the dorsal neurulation, considerable lateral tugging is felt on the right, due to the "short" nerve roots.

of the dura by adhesion bands, the careful cutting of which will liberate these roots from the dura (Fig. 36A, B), and magically "lengthen" them (Fig. 37) to allow for a comfortable neurulation (Figs. 38, 39).

It is evident from the above that leaving a sizable fringe or cuff of pia on each side while dissecting along the fusion line is of paramount importance, not only for providing a secure foothold for the micro-suturing, but a long fringe also endows a certain resilience to the imbricated seam. Just the opposite is seen when, very rarely, the axial profile of the "defatted" neural placode happens to have a bulky mid-sagittal peak resembling a tall pyramid with steep slopes (Fig. 40). The pial fringes in these cases are often vanishingly small, such that dorsal neurulation would require picking up the pia on the body of the folds and tying them together into 

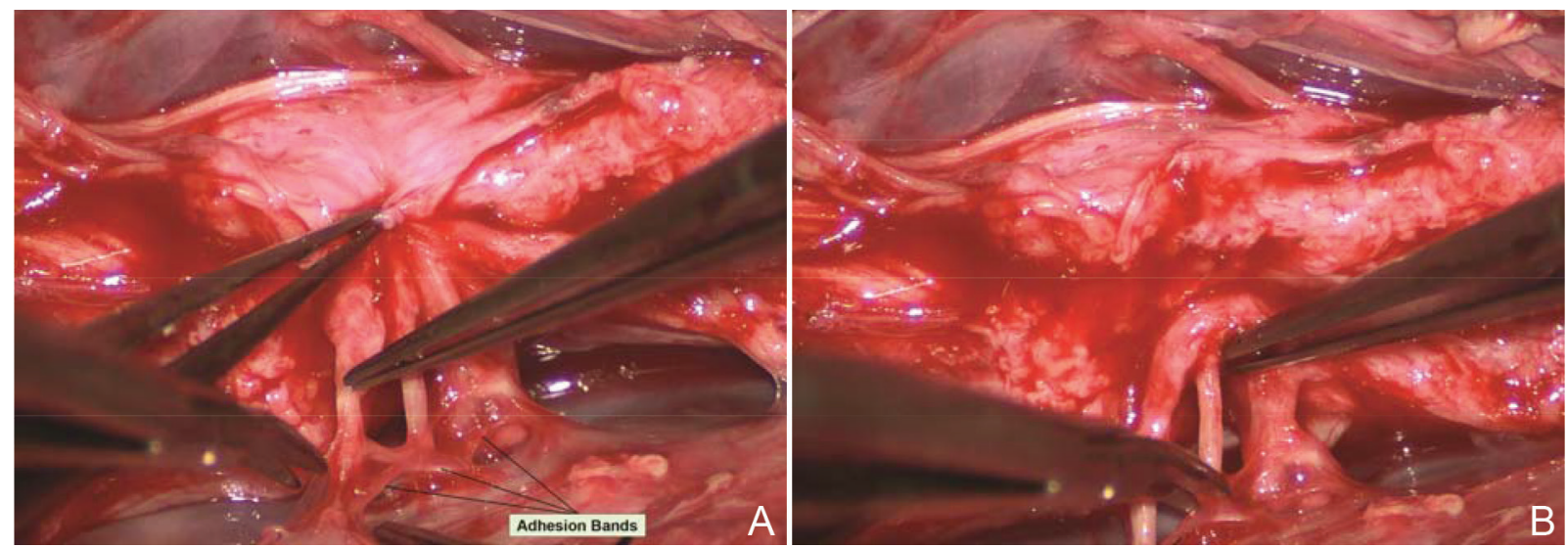

Fig. 36 Dealing with "short" nerve roots. A: The impression of shortness is spurious; these functional and supple roots appear short because they were bound tightly to the inner lining of the dura by adhesion bands that are being cut. B: More adhesion bands being cut.



Fig. 37 After having been detached from the inner lining of the dura, these "short" nerve roots become magically "lengthened."

a strangulating mess. The surgeon must learn to recognize the pyramid placode on the pre-operative axial MRI, and be prepared to exercise judgment whilst performing this critical part of placode reconstruction (Fig. 40B-D). Twice did I have to abandon neurulation because of adverse changes in the MEP due to the pyramid configuration, each time an agonizing chapter within the saga of lipoma resection.

After neurulation, the filum is usually cut for completeness.

\section{Step 5: Expansile Graft Duraplasty}

The argument for a graft dural closure comes from the belief that if the neurulated placode could slosh about in ample CSF within a capacious sac, the likelihood of re-attachment to the dura would be diminished. The choice of graft material is based on two important criteria: its texture and its propen- sity to leak CSF through the suture holes. Gortex is too stiff for infant dura and leaks badly, so was discarded in our early trials. CSF also regularly oozes through Duragen. Autologous fascia lata, once a popular option, is so soft and pliant that it swells and ebbs too lavishly with respiration and body movements, and during its in-phase almost completely collapses on to the neural placode. Thus we prefer the slightly full-bodied yet texturally compatible (with infant dura) bovine pericardium (Dura-Guard, Synovis Life Technologies Inc., St. Paul, Minnesota, USA) that can maintain a "puffedup" shape at all times and does not leak. A close second choice is Durepair (Medtronic Neurologic Technologies, Goleta, California, USA), made of reconstituted collagen matrix from bovine skin rather than straight bovine pericardium; it is only slightly stiffer than Dura-Guard and also does not leak at suture holes. The bovine graft is carefully measured and shaped to prevent having inward folds (Fig. 41). The opposing edges between the graft and the patient's dura are first lined up with judiciously placed 4-0 Surgilon sutures. Running prolene sutures are then used to achieve water-tight closure, always confirmed with Valsalva maneuvers each maintaining a pressure of 30-35 cm of water (depending on the patient's age) and sustained for 10 seconds (Fig. 42). A CSF pseudomeningocele compresses the dura against the neural placode and intuitively enhances re-tethering.

\section{Thoughts and Afterthoughts}

Our data show that total or near-total resection of spinal cord lipomas can be done in more than $90 \%$ of cases (Fig. 43). ${ }^{2)}$ In most instances, the 

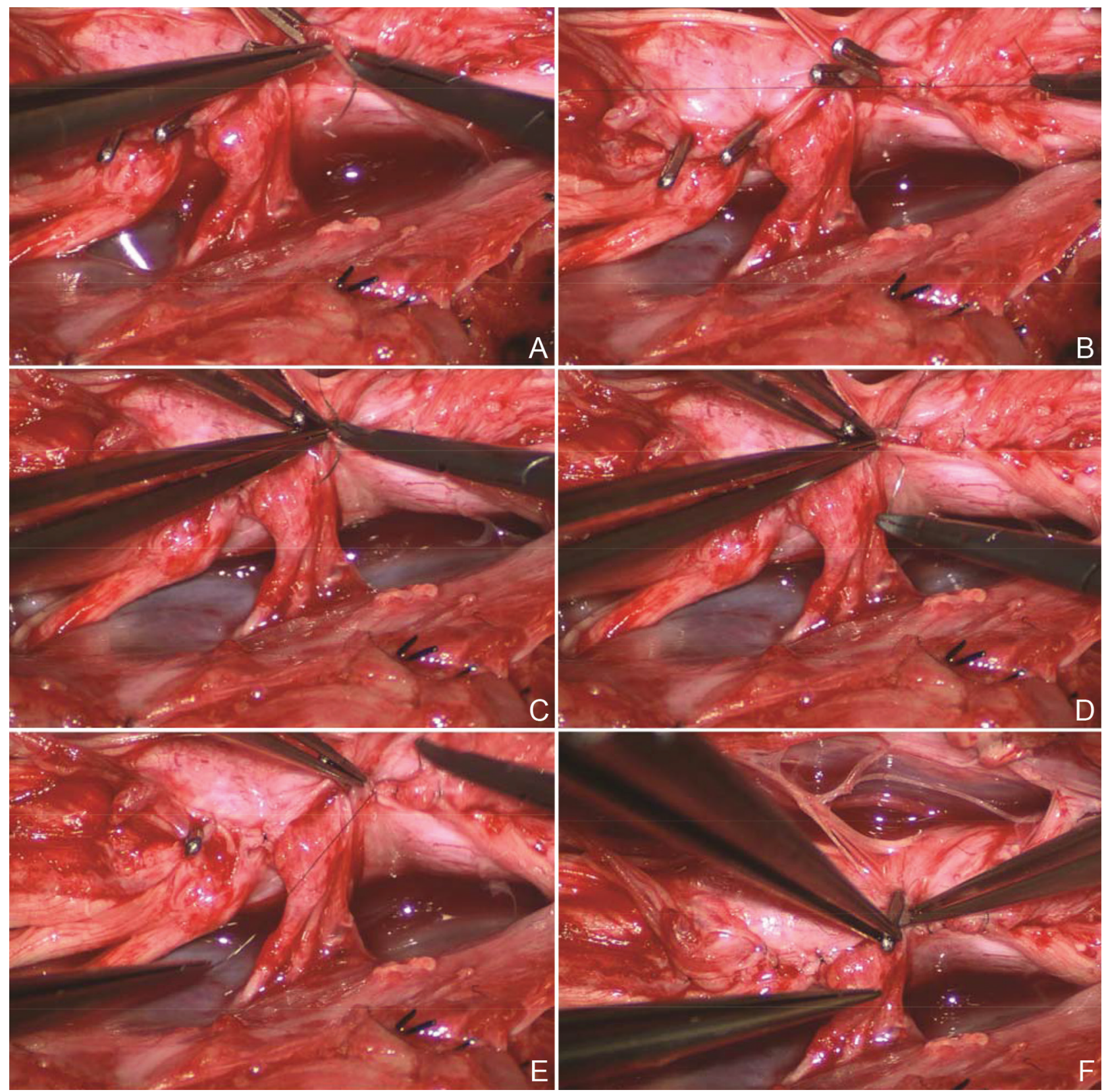

Fig. 38 Pia-to-pia neurulation of the neural placode. A: Pia-to-pia suturing with 8-0 nylon sutures with knots buried, B. Tying of micro sutures, apposing the pial fringes from each side of the placode, C: More 8-0 nylon suture, D: Yet more sutures, E: Burying the nylon knot, and F: Removing the last Weck clip.

small amount of residual fat is adherent to the DREZ and had been invaginated out of mischief during neurulation (Fig. 44). In the $8 \%$ of patients with unusually large amount of residual fat, the fat belongs to the ventral component of a chaotic lipoma and has been intentionally left pia-covered and therefore harmless.

To assess the "looseness" or degree of freedom of the reconstructed placode within the newly formed dural sac, we created the cord-sac ratio, defined as the ratio of the diameter of the cord to the diameter of the sac on the post-operative axial MRI at the bulkiest portion of the reconstructed segment. The ratios are grouped into low, less than $30 \%$, in the loosest sacs; medium, between $30 \%$ and $50 \%$, in moderately loose sacs; and high, greater than $50 \%$, in the tightest sacs (Fig. 45). In our total group of 315 patients, $227(72 \%)$ had low cord-sac ratios, $73(23.2 \%)$ had medium ratios, and only $15(4.8 \%)$ had high ratios. ${ }^{3)}$ 


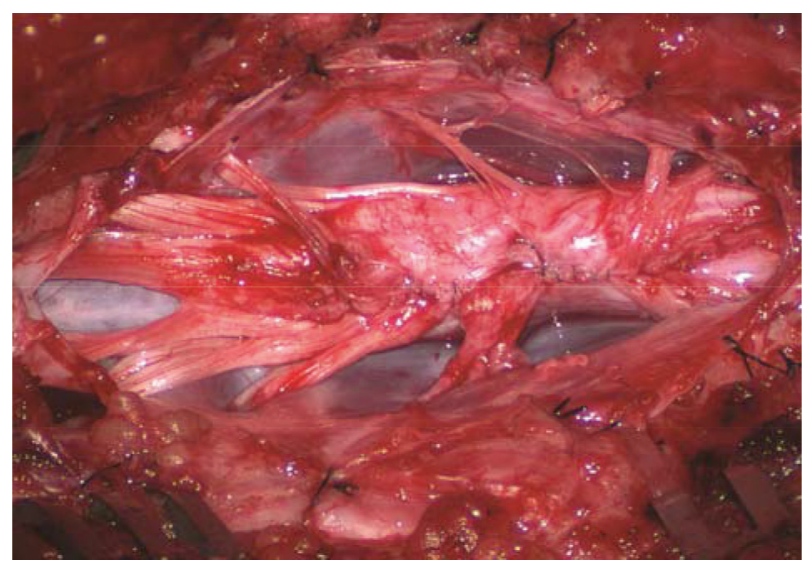

Fig. 39 Pia-to-pia neurulation completed with interrupted 8-0 nylon micro-sutures. The reconstituted neural tube is entirely pia-covered, with an unobtrusive dorsal seam. Note intact conus.

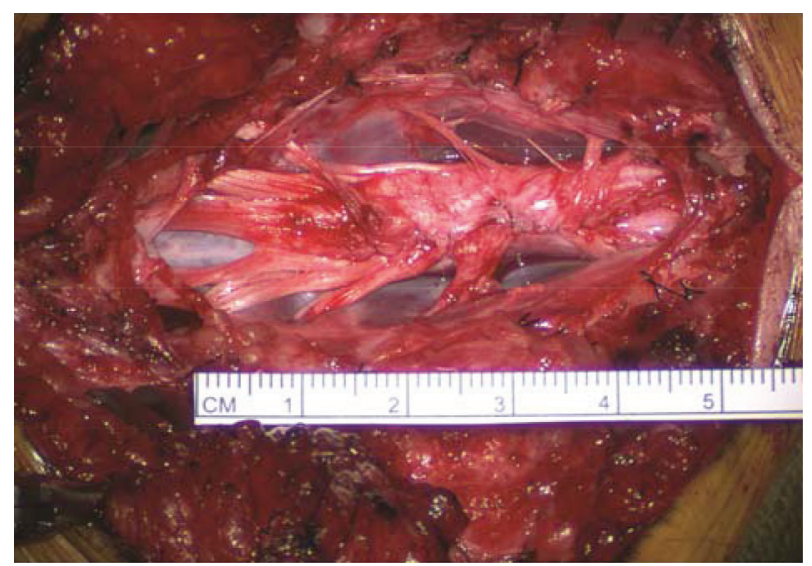

Fig. 41 Careful measurement of the length and width of the dural defect in preparation for fashioning the bovine pericardial graft.

Significance of the Pyramid Placode


Fig. 40 Dorsal lipoma with bulky pyramid placode-lesson learned. A: Note tall pyramid-shaped neural placode on axial profile, with steep side slopes interfacing with the lipoma. B: Lipoma stalk and dorsal lipoma. C: After lipoma resection, the placode is bulky in the middle and has thin pial fringes. D. Only the loose caudal part of the placode was neurulated. Rostral bulky part is left open because of TcMEP deterioration on forced attempts to suture. 


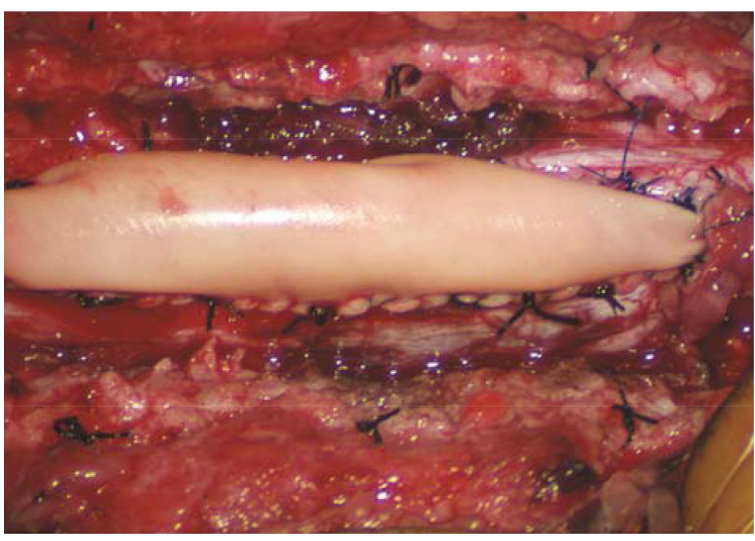

Fig. 42 After graft sutures are in place, water-tightness of the suture line is tested by several Valsalva maneuvers. An optimal graft is one that does not leak, puffs up with expiration, and registers no inward folding, as this one.

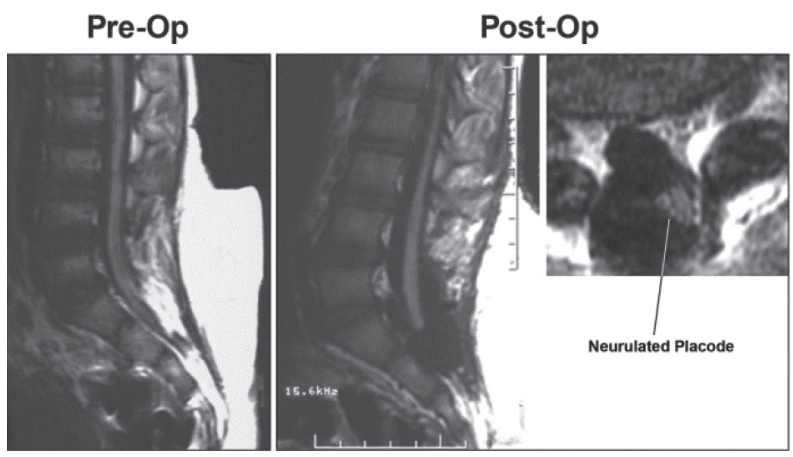

Fig. 43 Pre- and post-operative magnetic resonance imaging of a case of transitional lipoma with no residual fat after total lipoma resection. Note neurulated oblongshaped fat-free neural placode within a large dural sac.

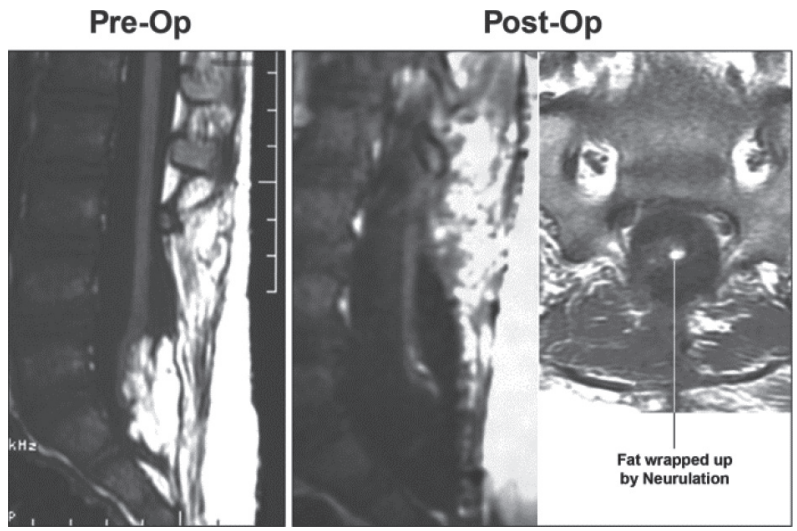

Fig. 44 Pre- and post-operative magnetic resonance imaging of a case of complex transitional lipoma with a very small amount $(<20 \mathrm{cu} \mathrm{mm})$ of residual fat after resection. Axial image shows the small round piece of fat is wrapped up within the roundly neurulated neural placode and therefore not exposed on the surface.


Fig. 45 Cord-sac ratios in the post-operative axial magnetic resonance imaging after total/near-total resection of lipoma. This ratio is obtained by dividing the sagittal diameter of the most bulbous portion of the post-neurulated neural placode by the sagittal diameter of the dural sac. In our series $72 \%$ have very loose sacs with cord-sac ratios $<30 \% ; 23.2 \%$ have intermediate ratios $30-50 \%$; and $4.8 \%$ have ratios $>\mathbf{5 0} \%$ with the least commodious cord-sac relationship. Cord-sac ratio estimates the degree of freedom of motion of the postneurulated spinal cord within its container sac.

Redo lesions are associated with a higher rate of residual fat and a higher cord-sac ratio. When the fat layer is infiltrated by heavy cicatrix from previous surgery, the cementing hold to the surrounding dura is much more tenacious and harder to detach, and the bright yellow of "virgin" fat is lost to a gray dense concretion much harder to distinguish from the white plane. The dissection often stops short of the white plane for fear of cutting too deep, and the result is a stiffer slab of residual scar-studded fat that not only augments the bulk of the placode, but also makes it awkward to fold at neurulation. The presence of this unyielding fat-scar at the DREZ often leads to "gouging," which may well be the cause of post-operative dysesthetic pain in redo patients. ${ }^{1)}$

Incomplete terminal untethering of the placode predictably ends in recurrence of symptoms. ${ }^{40-42)}$ We ascribe two explanations for the surgeon's hesitation to commit the final disconnecting cut. With very large transitional lipomas, the distal neural placode is buried in fat, and unless visualization is improved by substantial removal of fat, safe untethering cannot be done. Also, nerve twigs are sometimes seen issuing in pairs from the distal placode, making it seem impossible to complete the detachment without sacrificing functional cord. This assumption is spurious, for as long as two or three anal sphincter-activating roots, presumably $\mathrm{S}_{2}$ to $\mathrm{S}_{4}$, are identified and preserved, there should be no loss of function if the terminal cut is made just caudal to these roots. The small nerve twigs within the discarded stump that did not stimulate 
are probably coccygeal roots and therefore vestigial in human and have no essential function. ${ }^{1-3,43)}$

Thus, thorough lipoma and scar resection and terminal untethering impart the optimal bulk, texture, and maneuvrability to the neural placode for tensionless neurulation. A low post-operative cord-sac ratio has been shown to be the single most important factor in securing a long PFS in lipoma surgery $^{1,3)}$ (see below).

Finally, our experience unequivocally shows that chaotic lipomas are the most treacherous lesions. They can be recognized in the pre-operative MRI by the presence of ventral fat medial to the ventral nerve roots and by their association with sacral agenesis (Fig. 32). Compared to other lipoma types, chaotic lipomas are more likely to show conspicuous residual fat and high cord-sac ratio on the postoperative MRI. ${ }^{1-3)}$ The strategy for chaotic lesion is knowing just when to stop excavating deeper after the dorsal portion of the lipoma has been removed to enable neurulation. If the ventral fat is judged not amenable to total excision and neurulation, then its pial surface that had hitherto lain freely against the adjacent ventral dura should be left unsullied to avoid creating new adhesions (Fig. 32).

\section{Complications of Total Lipoma Resection}

Our combined neurological-urological deterioration rate following total/near-total resection is $4.1 \%,{ }^{3)}$ which compares favorably with rates in the literature ranging from $0.6 \%$ to $10 \%{ }^{6,7,42,44-56)}$ and averaging $3-7 \%$, all being associated with partial resection using more conservative techniques. Only $1.5 \%$ of our patients had new weakness but about $4 \%$ had neuropathic pain, ${ }^{1,3)}$ which, I suspect, is due to close encounter of the DREZ and dorsal roots with heat from the electrocautery. Most minor bleeding on the cord can be stopped with gentle tamponade and gelfoam, and if diathermy has to be applied, only the ultra-fine micro-tip irrigating bipolar cautery is used with very low current intensity.

The CSF leak rate $(0.7 \%)$ and wound complication $(1.1 \%)$ with total resection ${ }^{3)}$ are much lower than almost all of the published series (of partial resection), which record CSF leak rates from $2 \%$ to $47 \%^{7,42,45,46,49-51,54,56)}$ and wound dehiscence and infection rates of $2 \%$ to $26 \%{ }^{7,42,46,49,51,54,56,57)}$ Good results are owed to the following technical stipulations: (1) Enough bony exposure must be done caudally so that a cuff of healthy dura past the lowest extent of the lipoma can be made available for graft anastomosis. The graft should never be sewn to the web of fat at the remaining lipoma stump. Again, it needs to be pointed out here that the integrity of the caudal dura is seldom a problem with dorsal lipomas because a healthy conus (in dorsal lipoma) comes with respectable sacral dura whereas in many large transitional lipomas in which the conus is, by definition, part of the anomaly, the caudal dura can be forbiddingly shoddy. (2) Absolute water-tight closure of the graft with Prolene must be achieved, and challenged by Valsalva maneuver. (3) Synthetic or organic tissue glues are used if there is even a hint of a leak. (4) In large sacral lesions, there are often gaping muscle and fascial defects that cannot be primarily approximated. In these cases, paramedian relaxing incisions can be used on the flanking lumbodorsal fascia to facilitate sliding midline closure of the myofascial edges. (5) The large subcutaneous lipoma is never removed at the time of intraspinal surgery. The creation of this immense dead space will encourage collection of CSF which may turn into an enlarging pseudomeningocele that compresses the dural graft back on to the cord and prevents the desirable "billowing effect" of CSF on the new thecal sac. A tense pseudomeningocele may even threaten skin flap viability.

\section{Results of Total Resection}

The "why to operate" question is best answered by examining the mathematics of outcome between total resection and traditional treatment. We are in a rather unique position for this enterprise because the same senior surgeon (D.P.) performed the partial and the total resections, with the same technical style and rituals and unchanging adherence to details. A comparison between the early post-operative results of these two techniques and then their long-term outcomes is most revealing.

\section{Early Post-operative Results}

The early post-operative results are strikingly similar between our total and partial resection groups. ${ }^{11}$ For asymptomatic patients in both groups, the rates for neurological preservation are 98\% and 94\%, respectively. In symptomatic patients who had total or near total resection, $61 \%$ had normal or improved neurological status and 33\% remained unchanged, thus giving a rate of $94 \%$ for improvement or stabilization of disease. For symptomatic patients who had partial resection, 33\% were improved and $62 \%$ had disease stabilization, at least for the short term.

Our early post-operative results for total resection are thus comparable to or better than the literature, which mainly described partial resection. ${ }^{6,42,45,46,51,54,55)}$ In particular, prophylactic total resection of asymptomatic lipomas carries a small neurological risk when done 
in expert hands. Also, the benefits of radical surgery are obvious for patients with progressive deterioration. Finally, early improvement and stabilization rates are almost identical between total and partial resection, suggesting that the immediate benefits of surgery are due to the abrupt relief of traction on the conus and not to the extent of lipoma resection or placode reconstruction. Authors, who specifically described their inability to achieve complete disconnection of the conus from caudal attachment owing to the presence of obscuring residual fat and undelineated cord-lipoma margin, also pointed out the corresponding poor outcome of these patients. ${ }^{7,40,42)}$

When the post-operative status of patients with total resection is analyzed against pre-operative symptoms, the best result is seen with pain. Most of the sharp, dysesthetic leg and perineal pain will significantly diminish within 3 months, but not necessarily low back pain that is likely mechanical in origin. ${ }^{58,59)}$ Children also become more active and playful, and virtually never have chronic back complaints. Sensorimotor deficits also respond favorably with surgery. Although less than $20 \%$ of patients have actual normalization of motor function, the majority will substantially improve. ${ }^{1-3)}$ As with other forms of tethered cord, the milder and more recent deficits have better chance for good recovery. Bladder dysfunction responds favorably in about $20-30 \%$ of patients. ${ }^{2)}$ The subtype of neuropathic bladder with the best prognosis is the small capacity, spastic bladder with uninhibited detrusor contraction. Atonic bladders seldom improve with surgery, and intermittent catheterization usually needs to be continued indefinitely. The response of detrusor-sphincter dyssynergia to surgery is unpredictable. It is mandatory that cystometry and voiding cystourethrogram be repeated 3-6 months after surgery to determine what other urologic procedures such as bladder augmentation or ureteral conduits may be necessary to prevent reflux and frequent infections. Surgery has been known to arrest the rapid worsening of existing scoliosis in tethered cord. However, severe scoliosis still requires surgical realignment and fixation with instrumentation and fusion.

\section{Long-term Outcome}

Whilst the immediate benefits of total lipoma resection are due to the abrupt cessation of spinal cord tethering and are thus comparable to those from partial resection, ${ }^{2)}$ the long-term advantage of total over partial resection becomes very obvious if the PFSs of the two techniques are calculated for periods of 20 years. The PFS after total resection for all lipoma types and clinical subgroups is $88.1 \%$, vs. $34.6 \%$ for partial resection $^{3)}$ (Fig. 46). The differences in PFS are even

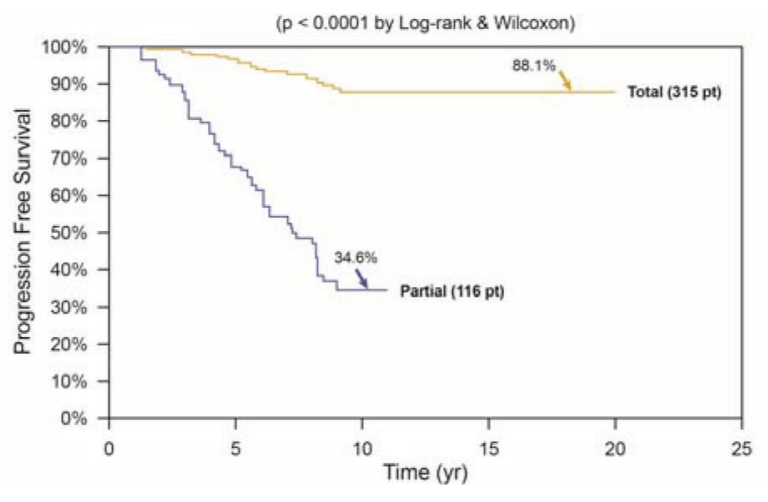

Fig. 46 Outcomes of total versus partial resection. Kaplan-Meier (KM) analysis for progression-free survival probability in total and partial resection of lipoma. The progression-free probability for total resection is $\mathbf{8 8 . 1} \%$ at 20 years, and $34.6 \%$ for partial resection at 10.5 years. The difference is highly significant $(p<0.0001$ by Log-rank and Wilcoxon). Note stabilization of disease after 8 years with total resection, but inexorable deterioration without disease arrest with partial resection. Partial: partial resection, Pt: patients, total: total resection.

more marked when the lipomas in each treatment group are segregated by the presence or absence of symptoms, age, and whether previous surgery was done. ${ }^{1,3)}$ The risk of symptomatic recurrence is 5.94 times higher with partial resection compared with total/near-total resection over 18 years by the Cox Proportional Hazard Regression Model, with a p value $<0.00001$. Even though the comparison is between prospective and retrospective data, the impressive difference in long-term PFS between the two techniques makes compelling argument for endorsing total/near-total over partial resection.

The question is often asked whether surgery should be offered to children with asymptomatic lipomas. In 2004, a prospective study of non-surgical management of asymptomatic lipomas from L'hôpital Necker-Enfants malades, Paris ${ }^{60)}$ reported an actuarial risk of deterioration of $33 \%$ over 9 years, with a PFS probability of $67 \% .{ }^{61)}$ A similar though retrospective study from London also reports a 10 year PFS of only $60 \%$ with non-surgical treatment, ${ }^{62)}$ with even lower PFSs when calculated for subgroups such as females, transitional lipomas and patients whose conus harbored a syrinx (Fig. 47). Given that children with lipomas have an actuarial life of 65-70 years, and given further that established neuropathic bladder and sensorimotor deficits seldom fully recover following post factum salvage surgery, ${ }^{42,44,51,54,56,63)}$ the endorsement for prophylactic surgery seems attractive if it does in fact offer clear-cut outcome advantage over conservative treatment. 


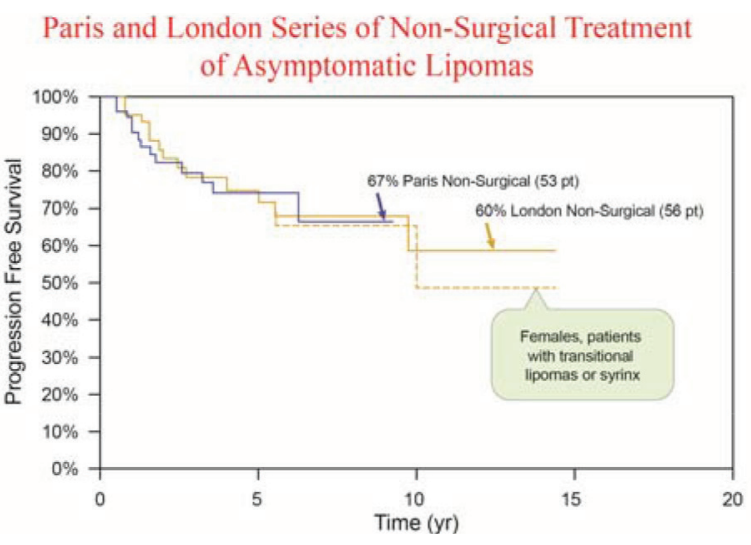

Fig. 47 Non-surgical treatment of asymptomatic lipomas. The blue line denotes the Paris series of 53 patients followed prospectively for 9 years, ${ }^{60)}$ with a PFS of $67 \%$. The yellow line denotes the retrospective London series of 56 patients followed for 10 years, ${ }^{62)}$ with a PFS of $60 \%$. In the London series, females, those with transitional lipomas and conus syrinx did worse, thus with an even worse PFS (dotted yellow line).

When the select group of young children without symptoms or prior lipoma surgery from our total resection series is put up against the Paris and London series, the superiority of total resection is obvious, with a 20 years PFS of $98.8 \%{ }^{3)}$ compared to $67 \%$ and $60 \%$ for conservative management, respectively ${ }^{60,62)}$ (Fig. 48). Furthermore, there is strong suggestion of stabilization of disease 5 years after total resection, whereas the probability of deterioration remains cumulative beyond 9 years with non-surgical treatment. ${ }^{60,62)}$ These robust statistics argue strongly for total resection, not only for symptomatic patients but also as prophylactic treatment for all children with lipomas.

There are currently no longitudinal data on untreated asymptomatic lipomas in adults, and the actuarial life span for adults is obviously shorter; so a forceful argument cannot be made for prophylactic surgery on asymptomatic adult lipomas. When a lipoma causes symptoms, however, it is widely assumed that deterioration would be inexorable, and aggressive surgery can be justified regardless of age.

\section{Cord-Sac Ratio and the Importance of Neurulation}

Comparing our two series of total and partial lipoma resections, we found a steep bias for low cord-sac ratios with total resection (Fig. 49), making cord-sac ratio the most likely factor that differentiates total from partial resection. That a low cord-sac ratio is

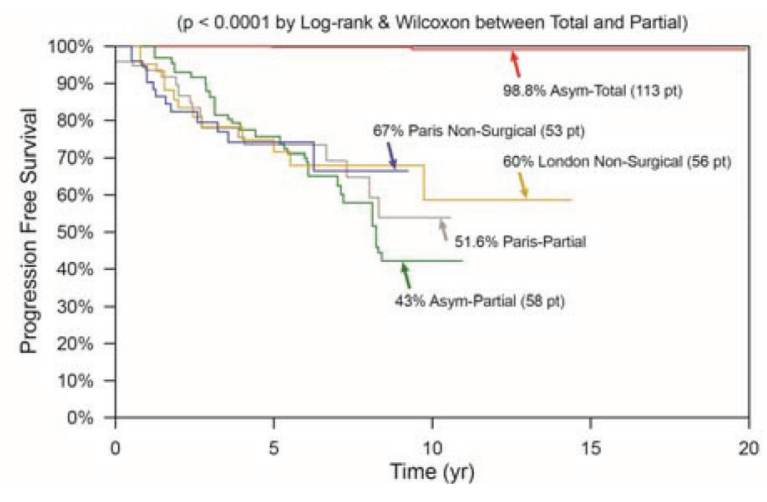

Fig. 48 Outcome differences among total resection, partial resection, and non-surgical management of asymptomatic virgin lipomas by Kaplan-Meier analysis. The non-surgical progressive-free survival graphs ("Paris Non-Surgical Asym") from the Parisian study ${ }^{60}$ and from the London study" ("London Non-Surgical Asym") are inserted only for visual comparison and not meant to imply a true "head-to-head" comparison. Progression-free probability at 20 years for the 113 asymptomatic virgin lipomas that had undergone total resection is $98.8 \%$, much better than $67 \%$ of the Parisian series or the $60 \%$ of the London series, and far superior to the $43.3 \%$ of our own partial resection series and the $51.6 \%$ of the Parisians' own partial resection series. The difference between total and partial resection for asymptomatic virgin lipomas is highly significant ( $p<0.0001)$. Asym-total: asymptomatic virgin lipomas treated by total resection, Asym-partial: asymptomatic virgin lipomas treated by partial resection, London non-surgical Asym: asymptomatic virgin lipomas from London, ${ }^{62)}$ pt: patients, Paris non-surgical Asym: asymptomatic virgin lipomas managed non-surgically in Paris. ${ }^{60)}$ The Paris partial resection and our own partial resection series are as labelled.

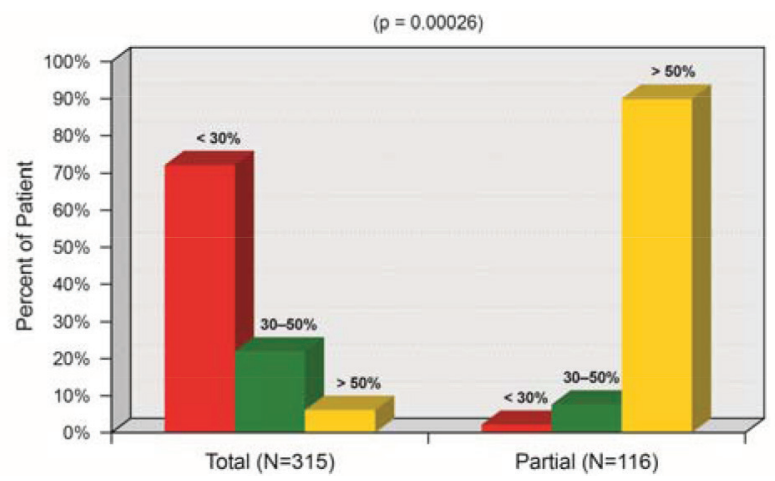

Fig. 49 Distribution of cord-sac ratios between the total resection and partial resection groups. Note $72 \%$ of patients who had total resection had cord-sac ratio $<30 \%$, versus less than $3 \%$ of patients who had partial resection. Conversely, only $6 \%$ of patients who had total resection had high ratio of $>50 \%$, versus over $90 \%$ of patients who had partial resection. The difference is significant $(p=0.00026)-<30 \% ; 30-50 \%$; and $>50 \%$ indicate the cord-sac ratios. 


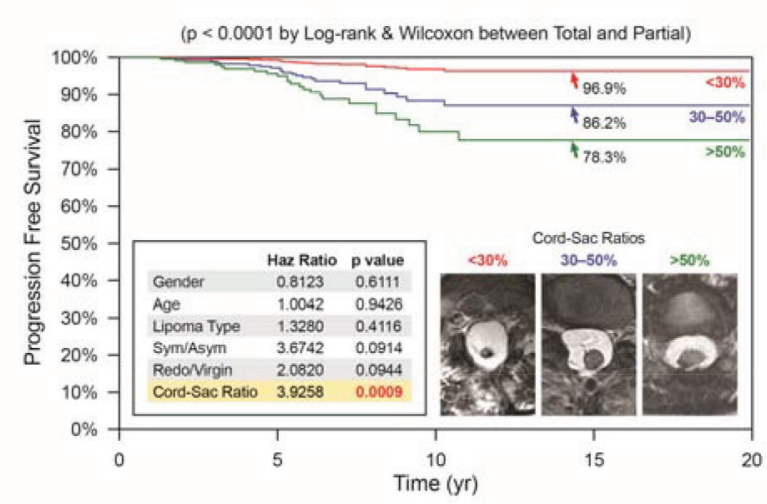

Fig. 50 Cox multivariate proportional hazard regression model analyzing the combined influence of six predictor variables (gender, age, lipoma type, symptoms, redo vs. virgin, and cord-sac ratio) on PFS after total resection, featuring the resultant effect of the three cord-sac ratios of $<30 \%, 30-50 \%$, and $>50 \%$. The hazard ratios and $p$ values for all six predictor variables are listed in the miniaturized table, showing that cord-sac ratio exerts the only significant independent influence on outcome. The respective progression-free probabilities, as indicated by the arrows, are $96.9 \%$ for low ratio, $86.2 \%$ for intermediate ratio, and $\mathbf{7 8 . 3} \%$ for high ratio. The differences in hazard prediction for the three ratios are highly significant $(p=0.0009$, in bold). $<30 \% ; 30-50 \%$, and $>50 \%$ indicate the three cord-sac ratios as shown in inset. Sym/Asym = symptomatic vs. asymptomatic lipomas.

an extremely important determinant of good outcome is amply supported by the Cox multivariate model, which shows that cord-sac ratio exerts a highly significant, independent influence on outcome. This means no matter how the associative influences of age, symptoms, lipoma types, and prior surgery reinforce or cancel each other, the influence of cord-sac ratio stands solitary and undiminished (Fig. 50). Thus, all other variables notwithstanding, a cord-sac ratio higher than $50 \%$ predicts a 3.9 times higher risk for disease progression than a low cord-sac ratio of less than $30 \%$, with a high statistical significance (Figs. 50, 51). The respective progression-free probabilities are $96.9 \%$ for low ratios less than $30 \%, 86.2 \%$ for intermediate ratios between $30 \%$ and $50 \%$, and $78.3 \%$ for ratios higher than $50 \%$.

In an operational sense, cord-sac ratio may be thought of as the summated product of the other predictors. For example, previous surgery on the lipoma undoubtedly makes it much harder to achieve a small cord-sac ratio: the texture and hue of scar tissue within the fat can be confused with the white plane, leading to retention of excessive fat and cicatrix and a bulky placode. ${ }^{2)}$ Since many
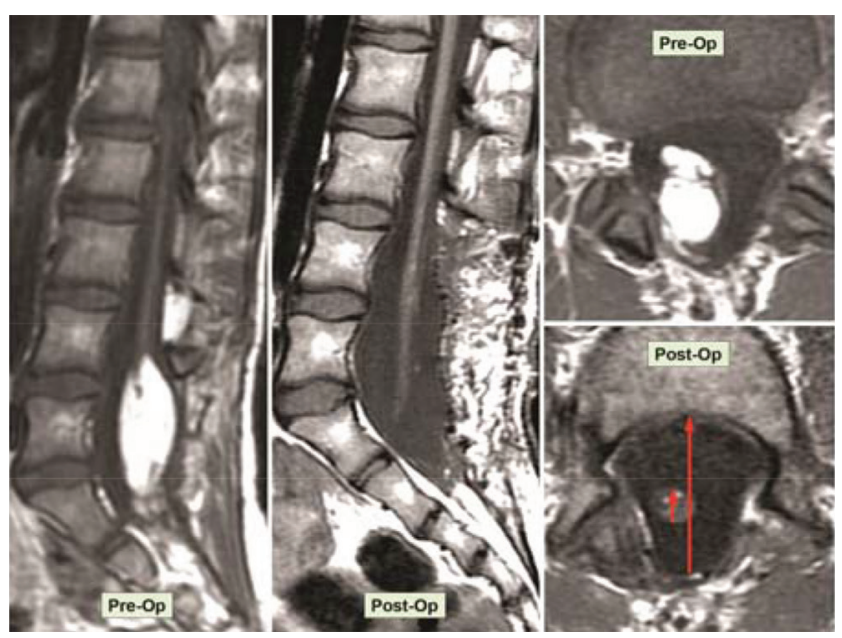

Fig. 51 The pre- and post-operative sagittal and axial magnetic resonance imaging of a 10 -year-old girl who has had two previous partial resections of a large transitional lipoma, and who developed new leg weakness. The achieved post-operative cord-sac ratio is $20 \%$ (lower right); and she enjoyed long-term progressionfree status.

of the symptomatic lipomas in our total resection group are redo lesions, their poor prognosis may in large part be due to the preeminence of the "redo factor." This "redo factor" is likely also the underlying cause for the negative effect of age on outcome, older patients are more likely to be symptomatic and to harbor redo lesions.

Though a loose fitting sac permits a greater freedom of cord motion within CSF and is proven important in preventing retethering, adhesion to the dura can still happen if what remains exposed is a sticky, unneurulated raw lipoma bed. Meticulous neurulation conceals this raw surface within an imbricated seam. Our experience of re-exploring unneurulated placodes leaves no doubt that this raw surface is a conspicuous focus of adhesion to the dura much more than a well-executed seam. Though not an easily quantifiable act, concealment of this adhesive surface through minutely careful neurulation must rank equally with a low cord-sac ratio as staunch insurance against retethering.

\section{Is Partial Resection Worse than No Resection?}

I have often been asked to revisit this issue and each time I feel slightly awkward that an affirmative answer seems self-serving in the present context of endorsing total resection. Yet it would be disingenuous to ignore the fact that our results for partial resection 
of 116 lipomas $^{1,2)}$ are clearly worse than the Paris and London series ${ }^{60,62)}$ of non-surgical treatment. Even if we select only our asymptomatic patients, which in essence eliminates all the surgically more treacherous redo lesions, the PFS for partial resection is still only $43 \%$ at 11 years $^{1)}$ compared to the $67 \%$ and $60 \%$ of the two non-surgical series ${ }^{3,60,62)}$; and Kulkarni et al.'s own partial resection series from Paris achieved only a slightly better PFS of $51 \%$ (Fig. 48). Other series of partial resection do not fare much better. If shorn of the terminal lipomas, Colak et al.'s series ${ }^{64)}$ would have a PFS of less than $50 \%$, and Pierre-Kahn's own series of partial resection would also have a lower than $40 \%$ PFS. ${ }^{7}$ Cochrane et al.'s ${ }^{45}$ transitional lesions had only $20 \%$ PFS over 10 years, and Cornette et al. ${ }^{65)}$ reported rapid progression of symptoms after partial resection.

We were impressed after having tackled more than 100 redo lipomas that the once abraded but unneurulated placode can be much more firmly fused to the dura by unyielding scar than an unchastened lipoma stalk. If the likelihood of deterioration had to do with the rigidity of transfixation of the cord, one would certainly expect partial resection to incur earlier and perhaps worse recurrence than if no surgery had been done, which was exactly the case in our recent analysis. ${ }^{3)}$ The time course of scar formation also explains why partial resection typically gives instantaneous relief of symptoms due to the initial untethering but is unable to sustain this effect in the long run because of delayed and worsening adhesions, producing an increasingly failing state that compares poorly with the natural history of the disease.

\section{The Dubious Rank of Chaotic Lipomas}

When the three different lipoma types (dorsal, transitional, and chaotic) were compared for outcome in our previous publication in 2010 featuring 238 total resections, there were no statistical differences among them. ${ }^{1)}$ However, even with the caveat of small number in the chaotic group (only 10 patients), the PFS curve for chaotic lesions ran considerably lower than the curves for dorsal and transitional lesions, giving the tentative impression that chaotic lipomas fared worse than the others.

In our recent publication of total resection comprising a larger population of 315 patients, ${ }^{3)}$ the number of chaotic lipomas has increased to 28. The PFSs for dorsal and transitional lesions remain statistically the same and also very similar

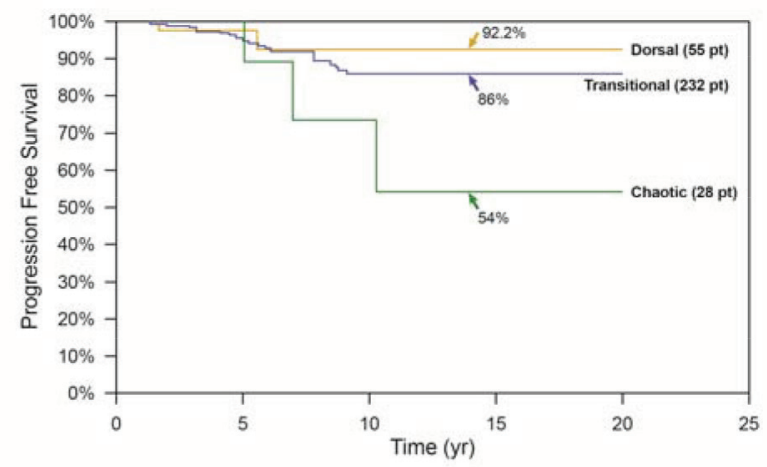

Fig. 52 The influence of lipoma type on outcome after total resection by Kaplan-Meier analysis. The progression-free probabilities for the three lipoma types are indicated by arrows. There is no significant difference in outcome between dorsal and transitional lipomas ( $p=0.458$ by Log-rank and $p=0.904$ by Wilcoxon) even after adjusting for sample size. There are "tentative" differences between chaotic and the other lipoma types when compared individually $(p=0.0472$ with dorsal lipomas and 0.0422 with transitional lipomas). Pt: patients.

to the 2010 figures $(92.2 \%$ and $86 \%$, respectively; $p=0.458$ by log rank), but the PFS of $54 \%$ for chaotic lesions has dropped further down from the other two curves (Fig. 52). Even though the difference between the outcomes of chaotic and the other two types of lipomas did only barely reach statistical significance ( $p$ value compared with dorsal lesions is 0.0492 and with transitional lesions is 0.0481), the "chaotic curve" is both visually and numerically nudging towards the categorical line that segregates out chaotic lipomas as a more sinister group. If one breaks down the chaotic group, one finds no patient with a cord-sac ratio less than $30 \%$. Even though the post-operative MRIs of chaotic lipomas may look better than the pre-operative images in showing less fat and slightly "looser" neural placode, the future is all too uncertain for many who are left with a high cord-sac ratio, an incompletely neurulated placode, and too much exposed, raw residual fat.

In Fig. 53, I inserted the London series on to the PFSs of the three lipoma types. The $54 \%$ PFS of chaotic lipomas looks uncomfortably similar to the $60 \%$ for non-surgical treatment from London. This begs the question whether asymptomatic chaotic lipoma should be treated prophylactically by any technique of resection. Hopefully, further accumulation of cases could put this nagging question to rest. 


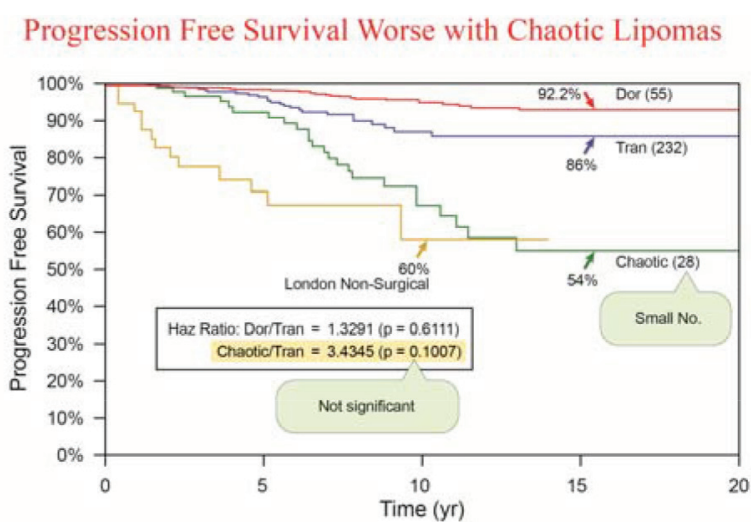

Fig. 53 The London non-surgical treatment progressionfree survival curve (PFS) ${ }^{62)}$ is inserted on to the three PFS curves of the different lipoma types obtained with the Cox proportional hazards analysis. The hazard ratio between chaotic and transitional lipomas is not statistically significant but that may be due to the small number in the chaotic group. The 10-year PFS from the London series is very similar to the PFS of the chaotic lipomas, which raises the question whether there is merit in resecting asymptomatic chaotic lipomas.

\section{Conclusion}

Total/near total resection of complex spinal cord lipomas and complete reconstruction of the neural placode produce a much better long-term PFS than partial resection. There are, in fact, strong indications that partial resection causes exuberant scarring at the lipoma-cord interface and consequently worsens prognosis compared to no surgery. In matching our results to published series of conservative treatment, we also find that total resection confers much greater benefits than non-surgical management in children with asymptomatic virgin lipomas. The post-operative neurological, urological, and wound complications for total resection are either comparable to or much lower than other series reporting on partial resection.

Multivariate analysis shows that a low post-operative cord-sac ratio and well executed neurulation of the neural placode strongly correlate with good long-term outcome. The ideal patient profile with early disease stabilization and the best recurrence-free survival has been identified to be the less than 2-year-old child who is without symptoms or history of prior surgery. This reinforces the notion that prophylactic total resection is the best option for the young child with an asymptomatic lipoma.

Based more on experience than hard statistics is the impression that chaotic lipomas are the most treacherous lesions to resect and may ultimately prove to be the most problematic lipoma type. If future data do not raise the PFS of chaotic lipomas a good distance above the curves for conservative treatment, then perhaps prophylactic surgery should not be offered to children with asymptomatic chaotic lesions.

Finally, it is perhaps apt to conclude the conclusion by supplying a few succinct answers to the three questions asked in the title of this review. The "how" of total resection, i.e., its technical package, can be learned by any neurosurgeon with a modicum of patience, tenacity, and open-mindedness. Many observers of the operation can attest that besides a few novel maneuvers and principles, the rest of the allegedly "unlearnable" conundrum of radical surgery is just basic microsurgical techniques. The intraoperative functional monitoring can also be done by any trained neurophysiologist.

As to the "why" and "when" questions, our two decades of data acquisition should have provided compelling reasons to recommend total resection for asymptomatic dorsal and transitional lipomas in children and for all symptomatic lipomas of all ages. The surgery should be done shortly after diagnosis, except in very young infants with stable neurology, for whom surgery is delayed till 6-months of age to minimize surgical morbidity.

\section{Conflicts of Interest Disclosure}

The author states that he has no conflicts of interests associated with the making of this manuscript.

\section{References}

1) Pang D, Zovickian J, Oviedo A: Long-term outcome of total and near-total resection of spinal cord lipomas and radical reconstruction of the neural placode, part II: outcome analysis and preoperative profiling. Neurosurgery 66: 253-272; discussion 272-273, 2010

2) Pang D, Zovickian J, Oviedo A: Long-term outcome of total and near-total resection of spinal cord lipomas and radical reconstruction of the neural placode: part I-surgical technique. Neurosurgery 65: 511-528; discussion 528-529, 2009

3) Pang D, Zovickian J, Wong ST, Hou YJ, Moes GS: Surgical treatment of complex spinal cord lipomas. Childs Nerv Syst 29: 1485-1513, 2013

4) Brunelle F, Sebag G, Baraton J, Carteret M, Martinat P, Pierre-Kahn A: Lumbar spinal cord motion measurement with phase-contrast MR imaging in normal children and in children with spinal lipomas. Pediatr Radiol 26: 265-270, 1996

5) Dick EA, de Bruyn R: Ultrasound of the spinal cord in children: its role. Eur Radiol 13: 552-562, 2003

6) Arai H, Sato K, Wachi, A: Surgical management in 81 patients with congenital intraspinal lipoma. Childs Nerv Syst 8: 171, 1992

7) Pierre-Kahn A, Zerah M, Renier D, Cinalli G, SainteRose C, Lellouch-Tubiana A, Brunelle F, Le Merrer M, 
Giudicelli Y, Pichon J, Kleinknecht B, Nataf F: Congenital lumbosacral lipomas. Childs Nerv Syst 13: 298-334, 1997

8) Dias M, Pang D: Human neural embryogenesis: A description of neural morphogenesis and a review of embryonic mechanisms, in Pang D (ed): Disorders of the Pediatric Spine. New York, Raven Press. 1994, pp 1-26

9) Hamilton HL, Boyd JD, Mossman HM: Human Embryology, ed 4. Baltimore, Williams \& Wilkins, 1972

10) Kunitomo K: The development and reduction of the tail and of the caudal end of the spinal cord. Contribut Embryol Carnegie Inst 8: 163-198, 1918

11) Streeter GL: Factors involved in the formation of the filum terminalis. Am J Anat 25: 1-12, 1919

12) Barson AJ: The vertebral level of termination of the spinal cord during normal and abnormal development. J Anat 106: 489-497, 1970

13) Jones PH, Love JG: Tight filum terminale. $A M A$ Arch Surg 73: 556-566, 1956

14) Caldarelli M, McLone DG, Colins JA, Suw J, Knepper PA: Vitamin A induced neural tube defects in a mouse. Concepts in Pediatr Neurosurg 6: 161-171, 1985

15) McLone DG, Suwa J, Collins JA, Poznaski S, Knepper PA: Neurulation: biochemical and morphological studies on primary and secondary neural tube defects. Concepts in Pediatr Neurosurg 4: 15-29, 1983

16) Marin-Padilla M: Clinical and experimental rachischisis, in Vinken PS, Bruyn GW (eds): Handbook of Clinical Neurology, vol 32. Amsterdam, NorthHolland, 1978, pp 159-191

17) Marin-Padilla M: Mesodermal alterations induced by hypervitaminosis A. J Embryol Exp Morphol 15: 261-269, 1966

18) Marin-Padilla M: Morphogenesis of anencephaly and related malformations. Curr Top Pathol 51: 145-174, 1970

19) Marin-Padilla M, Marin-Padilla TM: Morphogenesis of experimentally induced Arnold-Chiari malformation. J Neurol Sci 50: 29-55, 1981

20) Marin-Padilla M: Morphogenesis of experimental encephalocele (Cranioschisis occulta). J Neurol Sci 46: 83-99, 1980

21) Marin-Padilla M: Notochordal-basichondrocranium relationships: abnormalities in experimental axial skeletal (dysraphic) disorders. J Embryol Exp Morphol 53: 15-38, 1979

22) Marin-Padilla M: The tethered cord syndrome: developmental considerations, in Holtzmann RNN, Stein BM (eds): The Tethered Spinal Cord. New York, Thieme, 1985, pp 3-13

23) McLone DG, Knepper PA: Role of complex carbohydrates and neurulation. Pediatr Neurosci 1: 2-9, 1986

24) Morriss-Kay GM, Crutch B: Culture of rat embryos with beta-D-xyloside: evidence of a role for proteoglycans in neurulation. J Anat 134: 491-506, 1982
25) O’Shea KS, Kaufman MH: Phospholipase C-induced neural tube defects in the mouse embryo. Experientia 36: 1217-1219, 1980

26) Toole BP: Glycosaminoglycans in morphogenesis, in Hay E (ed): Cell Biology of Extracellular Matrix. New York, Plenum Press, 1981, pp 229-294

27) Detwiler SR, Holtzer H: The inductive and formative influence of the spinal cord upon the vertebral column. Bull Hosp Joint Dis 15: 114-123, 1954

28) Källén B: Early embryogenesis of central nervous system with special reference to closure defects. Dev Med Child Neurol 16(Suppl): 16:44+, 1968

29) McLone DG, Naidich TP: Spinal dysraphism: experimental and clinical, in Holtzman RN, Stein BM (eds): The Tethered Spinal Cord. New York, Thieme-Stratton, 1985

30) Pang D: Spinal cord lipomas, in Pang D (ed): Disorders of the Pediatric Spine. New York, Raven Press, 1995, pp 175-201

31) Pang D: Spinal cord lipoma, in Batjer H, Loftus C (eds): Textbook of Neurological Surgery. New Jersey, Lippincott, Williams and Wilkins, 2002

32) Pang D: Tethered cord syndrome, in Hoffman HJ (ed): Advances in Pediatric Neurosurgery. Philadelphia, Hanley and Belfus Inc., 1986, pp 45-79

33) Schoenwolf GC: Histological and ultrastructural studies of secondary neurulation in mouse embryos. Am J Anat 169: 361-376, 1984

34) Müller F, O'Rahilly R: The development of the human brain, the closure of the caudal neuropore, and the beginning of secondary neurulation at stage 12. Anat Embryol (Berl) 176: 413-430, 1974

35) O'Rahilly R, Meyer DB: The timing and sequence of events in the development of the human vertebral column during the embryonic period proper. Anat Embryol (Berl) 157: 167-176, 1979

36) Schoenwolf GC: Histological and ultrastructural observations of tail bud formation in the chick embryo. Anat Rec 193: 131-147, 1979

37) Pang D: Electrophysiological monitoring for tethered cord surgery, in Yamada S (ed): Tethered Cord Syndrome, ed 2. New York, Thieme Medical Publishers, 2010, pp 199-209

38) Pang D, Casey K: Use of an anal sphincter pressure monitor during operations on the sacral spinal cord and nerve roots. Neurosurgery 13: 562-568, 1983

39) Pang D: Intraoperative neurophysiology of the conus medullaris and cauda equina. Childs Nerv Syst 26: 411-412, 2010

40) Chapman PH, Davis KR: Surgical treatment of spinal lipomas in childhood. Pediatr Neursurg 19: 267-275; discussion 274-4, 1993

41) Pierre-Kahn A, Lacombe J, Pichon J, Giudicelli Y, Renier D, Sainte-Rose C, Perrigot M, Hirsch JF: Intraspinal lipomas with spina bifida. Prognosis and treatment in 73 cases. J Neurosurg 65: 756-761, 1986

42) Xenos C, Sgouros S, Walsh R, Hockley A: Spinal lipomas in children. Pediatr Neurosurg 32: 295-307, 2000

Neurol Med Chir (Tokyo) 55, September, 2015 
43) Chapman PH: Congenital intraspinal lipomas: anatomic considerations and surgical treatment. Childs Brain 9: 37-47, 1982

44) Atala A, Bauer SB, Dyro FM, Shefner J, Shillito J, Sathi S, Scott RM: Bladder functional changes resulting from lipomyelomeningocele repair. J Urol 148(2 Pt 2): 592-594, 1992

45) Cochrane DD, Finley C, Kestle J, Steinbok P: The patterns of late deterioration in patients with transitional lipomyelomeningocele. Eur J Pediatr Surg 10(Suppl 1): 13-17, 2000

46) Hoffman HJ, Taecholarn C, Hendrick EB, Humphreys RP: Management of lipomyelomeningoceles. Experience at the Hospital for Sick Children, Toronto. J Neurosurg 62: 1-8, 1985

47) James CC, Lassman LP: Diastematomyelia and the tight filum terminale. J Neurol Sci 10: 193-196, 1970

48) James HE, Canty TG: Human tails and associated spinal anomalies. Clin Pediatr (Phila) 34: 286-288, 1995

49) Kanev PM, Lemire RJ, Loeser JB, Berger MS: Management and long-term follow-up review of children with lipomyelomeningocele, 1952-1987. J Neurosur 73: 48-52, 1990

50) Koyanagi I, Iwasaki Y, Hida K, Abe H, Isu T, Akino M: Surgical treatment supposed natural history of the tethered cord with occult spinal dysraphism. Childs Nerv Syst 13: 268-274, 1997

51) La Marca F, Grant JA, Tomita T, McLone DG: Spinal lipomas in children: outcome of 270 procedures. Pediatr Neurosurg 26: 8-16, 1997

52) McGuire EJ: The innervation and function of the lower urinary tract. J Neurosurg 65: 278-285, 1986

53) McGuire EJ, Woodside JR, Borden TA, Weiss RM: Prognostic value of urodynamic testing in myelodysplastic patients. J Urol 126: 205-209, 1981

54) McLone DG, Naidich TP: Laser resection of fifty spinal lipomas. Neurosurgery 18: 611-615, 1986

55) Sathi S, Madsen JR, Bauer S, Scott RM: Effect of surgical repair on the neurologic function in infants with lipomeningocele. Pediatr Neurosurg 19: 256-259, 1993
56) Sutton LN: Lipomyelomeningocele. Neurosurg Clin N Am 6: 325-338, 1995

57) James CCM, Williams J, Brock W, Kaplan GW, U HS: Radical removal of lipomas of the conus and cauda equina with laser microsurgery. Neurosurgery 15: $340-345,1984$

58) Hoffman HJ, Hendrick EB, Humphreys RP: The tethered spinal cord: its protean manifestations, diagnosis and surgical correction. Childs Brain 2: 145-155, 1976

59) Pang D, Wilberger JE: Tethered cord syndrome in adults. J Neurosurg 57: 32-47, 1982

60) Kulkarni AV, Pierre-Kahn A, Zerah M: Conservative management of asymptomatic spinal lipomas of the conus. Neurosurgery 54: 868-873; discussion 873-875, 2004

61) Kulkarni HV, Pierre-Kahn A, Zerah M: Conservative management of asymptomatic spinal lipomas of the conus. Neurosurgery 54: 868-873; discussion 873-875, 2004

62) Wykes V, Desai D, Thompson DN: Asymptomatic lumbosacral lipomas-a natural history study. Childs Nerv Syst 28: 1731-1739, 2012

63) Byrne RW, Hayes EA, George TM, McLone DG: Operative resection of 100 spinal lipomas in infants less than 1 year of age. Pediatr Neurosurg 23: 182-186; discussion 186-187, 1995

64) Colak A, Pollack IF, Albright AL: Recurrent tethering: a common long-term problem after lipomyelomeningocele repair. Pediatr Neurosurg 29: 184-190, 1998

65) Cornette L, Verpoorten C, Lagae L, Plets C, Van Calenbergh F, Casaer P: Closed spinal dysraphism: a review on diagnosis and treatment in infancy. Eur J Paediatr Neurol 2: 179-185, 1998

Address reprint requests to: Dachling Pang, MD, FRCS(C), FACS, Department of Pediatric Neurosurgery, Kaiser Permanente Medical Center, 3600 Broadway Boulevard, 3rd Floor, Suite 39, Oakland, California 94611, USA. e-mail: PangTV@aol.com 